\title{
Aluminum Tralen Complex Meditated Reversible-Deactivation Radical Polymerization of Vinyl Acetate
}

Shih-Ji Chen ${ }^{\dagger}$, Shan-Cheng Tang ${ }^{\dagger}$, Pan Zhang ${ }^{\ddagger}$, Changle Chen*,‡, and Chi-How Peng*, $\dagger$

†Department of Chemistry and Frontier Research Center on Fundamental and Applied Sciences of Matters, National Tsing Hua University, Hsinchu, 30013, Taiwan

$\$$ CAS Key Laboratory of Soft Matter Chemistry, iChEM (Collaborative Innovation Center of Chemistry for Energy

Materials), Department of Polymer Science and Engineering, University of Science and Technology of China, Hefei 230026, People's Republic of China

\section{Contents}

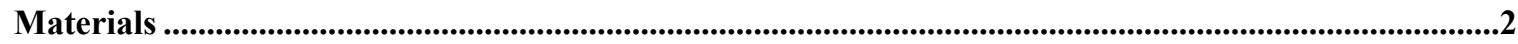

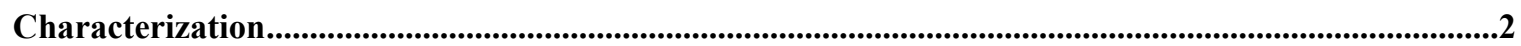

General procedure for polymerization n..............................................................................................3

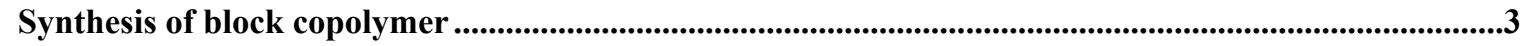

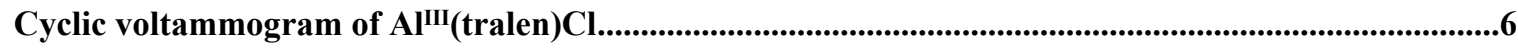

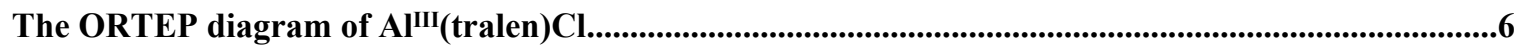

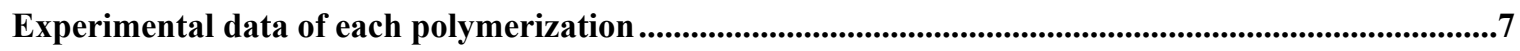

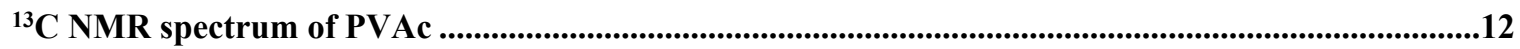

${ }^{1}$ H NMR and DOSY-NMR spectrum of PVAc- $b$-PNVP block copolymer .......................................17

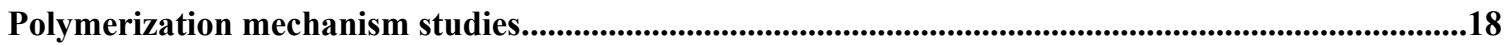

Analysis for the structure of the $\omega$ chain ends ..................................................................................18

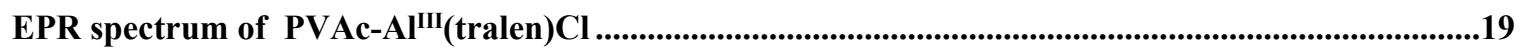

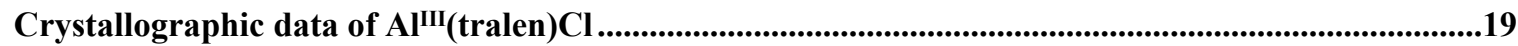




\section{Materials}

Toluene was dried by refluxing at least $24 \mathrm{~h}$ over $\mathrm{CaH}_{2}$. Vinyl acetate ( $99 \%$, Merck) was degassed by three free-pump-thaw cycles and distilled under reduced pressure. Methyl acrylate ( $98 \%$, ACROS), $N$-vinylpyrrolidone ( $98 \%$, ACROS), acrylonitrile ( $99 \%$, ACROS), tert-butyl acrylate ( $99 \%$, ACROS), and styrene ( $99 \%$, Aldrich) were passed through the basic $\mathrm{Al}_{2} \mathrm{O}_{3}$ to remove the inhibitor. Dimethylformamide (DMF, Alfa Aesar), 2,2'-azo-bisisobutyronitrile (AIBN, Aencore), and 1,1'azobis(cyclohexanecarbonitrile) (ABCN, Aldrich) were used without any purification. Deuterated solvents (Aldrich) were dried over molecular sieves.

\section{Characterization}

All manipulations were carried out under a dry nitrogen atmosphere using an MBraun glove box and Schlenk flask. NMR spectra were recorded by Mercury $400 \mathrm{MHz}$ and $500 \mathrm{MHz}$ Spectrometer. ${ }^{1} \mathrm{H}$ NMR chemical shifts are given in ppm versus residual protons in deuterated solvents as follows: $\delta 7.24 \mathrm{ppm}\left(\mathrm{CDCl}_{3}\right) \cdot{ }^{13} \mathrm{C}$ NMR chemical shifts are given in ppm versus residual ${ }^{13} \mathrm{C}$ in solvents as follows: $\delta 77 \mathrm{ppm}\left(\mathrm{CDCl}_{3}\right)$. NMR spectroscopy was used to identify the ligand structure and monomer conversion. Polymer products of PVAc, PMA, P $t$ BA and PSt were characterized by a gel permeation chromatography (GPC) system equipped with three Shodex columns (Shodex KF-802, Shodex KF-803, and Shodex KF-805) using THF eluent at $30{ }^{\circ} \mathrm{C}$ and $1.0 \mathrm{~mL} \mathrm{~min}{ }^{-1}$ flow rate. The signal was collected by DIONEX Shodex RI-101 refractometer (RI) detector. The molecular weight was calibrated with narrow linear poly(styrene) Shodex standard (SM-105) ranging in molecular weight from $1.20 \times 10^{2} \mathrm{~g} \mathrm{~mol}^{-1}-2.61 \times 10^{6} \mathrm{~g} \mathrm{~mol}^{-1}$. Polymer products of PNVP and PAN were were characterized by a gel permeation chromatography (GPC) system equipped with three Waters columns (Styragel ${ }^{\circledR}$ HR 2 DMF, Styragel ${ }^{\circledR}$ HR 4 DMF, and Styragel ${ }^{\circledR}$ HR 5 DMF) using DMF with $\operatorname{LiBr}(0.1 \mathrm{M})$ as the eluent at $80{ }^{\circ} \mathrm{C}$ with flow rate equal of $1 \mathrm{~mL} \mathrm{~min}^{-1}$ and Refractive Index Detector (RI-101, Thermo Scientific Dionex). The calibration was based on linear poly(ethylene glycol) (PEG) with molecular weight as $2000,5000,17800,31400$, and $72750 \mathrm{~g} / \mathrm{mol}$. Molecular weights and the $M_{\mathrm{w}} / M_{\mathrm{n}}$ values were calculated using DIONEX Chromeleon software. EPR measurements were performed at the X-band using a Bruker E580 spectrometer equipped with a Bruker ELEXSYS super-high-sensitivity cavity. The cyclic voltammetry (CV) was measured by three electrode potentiostat (CH Instruments, Model 611E) in $\mathrm{TBAPF}_{6} / \mathrm{DMSO}(0.1 \mathrm{M})$ using a Pt wire as the working electrode. The potentials were measured under scan rate $=0.1 \mathrm{~V} \mathrm{~s}^{-1}$ at $298 \mathrm{~K}$ with ferrocene as an internal standard.

\section{Synthesis of N, N'-di(cyclohepta-2,4,6-trien-1-one-2-yl)-1,2- diaminobenzene}

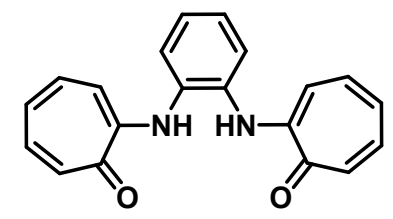

To a mixture of tris(dibenzylideneacetone)dipalladium $\left(\mathrm{Pd}_{2}(\mathrm{dba})_{3}\right)(45.8 \mathrm{mg}, 0.05 \mathrm{mmol})$, 1,1'-Binaphthalene-2,2'diylbis(diphenylphosphane) (rac-BINAP) $(62.3 \mathrm{mg}, 0.1 \mathrm{mmol}), \mathrm{Cs}_{2} \mathrm{CO}_{3}(4.56 \mathrm{~g}, 14 \mathrm{mmol})$, 2-triflatotropone (4.4 g, $\left.24 \mathrm{mmol}\right)$ and $o$-phenylenediamine $(1.08 \mathrm{~g}, 10 \mathrm{mmol})$ were added in toluene $(50 \mathrm{~mL})$. The mixture was stirred at $100{ }^{\circ} \mathrm{C}$ for $24 \mathrm{~h}$ under nitrogen. After being cooled to room temperature, the insoluble solid was filtered through celite, the filtrate was evaporated under reduced pressure. The purified product as yellow solid was obtained by the chromatography with EtOAc, and the yield was $74.9 \%$. ${ }^{1} \mathrm{H}$ NMR $\left(400 \mathrm{MHz}, \mathrm{CDCl}_{3}\right) \delta(\mathrm{ppm}): 8.53(\mathrm{~s}, 2 \mathrm{H}, \mathrm{NH}), 7.51-7.43(\mathrm{~m}, 2 \mathrm{H}), 7.38-7.31(\mathrm{~m}, 2 \mathrm{H}), 7.26(\mathrm{dd}, J=11.5$, $8.3 \mathrm{~Hz}, 2 \mathrm{H}), 7.16(\mathrm{~d}, J=11.7 \mathrm{~Hz}, 2 \mathrm{H}), 7.05(\mathrm{t}, J=10.2 \mathrm{~Hz}, 2 \mathrm{H}), 6.85(\mathrm{~d}, J=10.2 \mathrm{~Hz}, 2 \mathrm{H}), 6.74(\mathrm{t}, J=9.4 \mathrm{~Hz}, 2 \mathrm{H}) .{ }^{13} \mathrm{C}$ NMR $\left(101 \mathrm{MHz}, \mathrm{CDCl}_{3}\right) \delta(\mathrm{ppm}): 177.18,152.50,137.57,135.63,133.23,131.36,127.14,126.27,125.34,110.95$. HRMS $(\mathrm{m} / \mathrm{z})$ : calcu. $\mathrm{C}_{20} \mathrm{H}_{16} \mathrm{~N}_{2} \mathrm{O}_{2}: 316.1212$, found: $317.1276[\mathrm{M}+\mathrm{H}]^{+}$. 


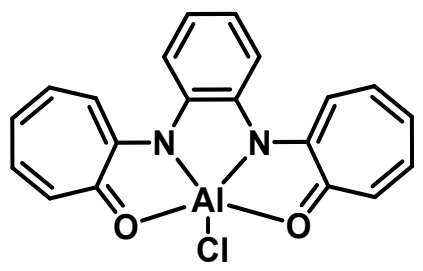

2,2'-(1,2-phenylenebis(azan-ediyl))bis(cyclohepta-2,4,6-trien-1-one (1.58 g, $5 \mathrm{mmol}$ ) was dissolved in toluene $(25 \mathrm{~mL})$, and diethylaluminum chloride $(4.5 \mathrm{~mL}, 5 \mathrm{mmol}, 0.9 \mathrm{M}$ in toluene) was added in the solution at room temperature. The mixture was stirred at room temperature for $2 \mathrm{~h}$, then stirred at $100^{\circ} \mathrm{C}$ for another $12 \mathrm{~h}$. After cooling down to room temperature, the yellow solid was filtered and washed with hexane $(5 \mathrm{~mL})$ three times in the glove box. The product $\mathrm{Al}^{\mathrm{III}}($ tralen$) \mathrm{Cl}$ was dried under reduced pressure (1.78 g, yield as 95\%). ${ }^{1} \mathrm{H}$ NMR $\left(400 \mathrm{MHz}, \mathrm{CDCl}_{3}\right) \delta(\mathrm{ppm}): 8.00(\mathrm{~d}, J=11.4 \mathrm{~Hz}, 2 \mathrm{H}), 7.84-7.78(\mathrm{~m}, 2 \mathrm{H})$, 7.55-7.44 (m, 6H), $7.31(\mathrm{~m}, 2 \mathrm{H}), 7.04(\mathrm{t}, J=8.8 \mathrm{~Hz}, 2 \mathrm{H}) .{ }^{13} \mathrm{C}$ NMR $\left(101 \mathrm{MHz}, \mathrm{CDCl}_{3}\right) \delta(\mathrm{ppm}):$ 171.96, 158.14, 139.29, 139.19, 137.42, 127.54, 125.47, 123.61, 119.77, 119.17. MALDE-TOF-MS (m/z): Calcu. $\mathrm{C}_{20} \mathrm{H}_{14} \mathrm{AlClN}_{2} \mathrm{O}_{2}[\mathrm{M}]: 376.0559$; Calcu. $\mathrm{C}_{20} \mathrm{H}_{14} \mathrm{AlN}_{2} \mathrm{O}_{2}[\mathrm{M}-\mathrm{Cl}]^{+}: 341.0871$, Found : 341.0975 [M-Cl] .

\section{General procedure for polymerization}

AlII(tralen)Cl (28.6 mg, $0.077 \mathrm{mmol}), \mathrm{NaBArF}(68.23 \mathrm{mg}, 0.077 \mathrm{mmol})$, and AIBN (249 mg, $1.5 \mathrm{mmol})$ were placed in a 50 $\mathrm{mL}$ Schlenk flask and degassed by three vacuum/nitrogen cycles. Dry, degassed vinyl acetate $(7 \mathrm{~mL}, 77 \mathrm{mmol})$ was added by syringe under nitrogen. The mixture was stirred at $30{ }^{\circ} \mathrm{C}$ for $10 \mathrm{~min}$, then heated at $60{ }^{\circ} \mathrm{C}$ to start the polymerization. The monomer conversion was determined by ${ }^{1} \mathrm{H}$ NMR spectrum. The polymerizations of methyl acrylate, $N$-vinylprrolidone, acrylonitrile, tert-butyl acrylate, and styrene were performed with the similar procedure.

\section{Synthesis of block copolymer}

Macroinitiator of PVAc-Al ${ }^{\mathrm{III}}($ tralen $) \mathrm{BArF}\left(M_{\mathrm{n}}=8000, \mathrm{PDI}=1.40\right)$ was prepared by the general polymerization procedure as $\left[\mathrm{Al}^{\mathrm{III}}(\text { tralen }) \mathrm{Cl}\right]_{0} /[\mathrm{NaBArF}]_{0} /[\mathrm{AIBN}]_{0} /[\mathrm{VAc}]_{0}=1 / 1 / 20 / 200$ at $60^{\circ} \mathrm{C}$ in bulk, and unreacted monomers were removed after the polymerization by vacuum. The flask with macroinitiator was then filled with nitrogen, followed by the addition of DMF $(4 \mathrm{~mL})$ and $N$-vinylprrolidone $(2 \mathrm{~mL}, 18 \mathrm{mmol})$. The mixture was stirred and heated at $60{ }^{\circ} \mathrm{C}$ to start the polymerization.

\section{NMR spectrum for tralen $\mathrm{H}_{2}$ and $\mathrm{AI}^{\mathrm{III}}($ tralen$) \mathrm{Cl}$}
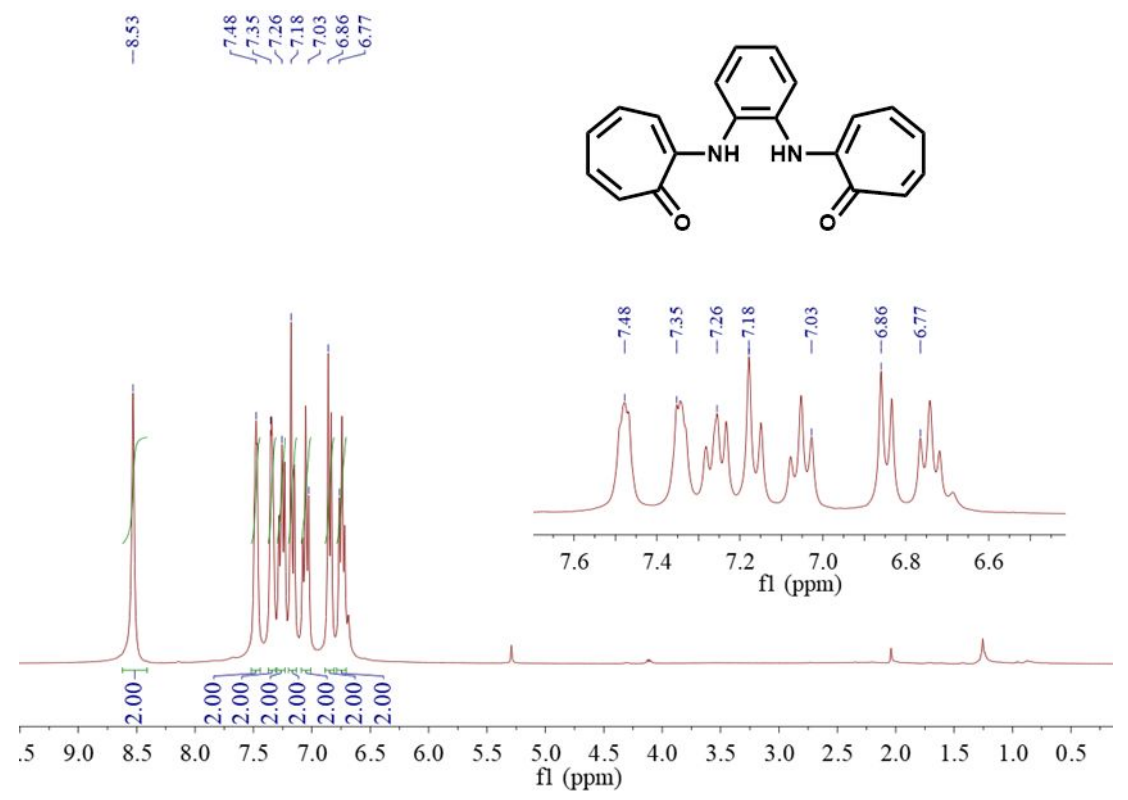

Figure S1. ${ }^{1} \mathrm{H}$ NMR spectrum of 2,2'-(1,2-phenylenebis(azan-ediyl))bis(cyclohepta-2,4,6-trien-1-one) in $\mathrm{CDCl}_{3}$. 

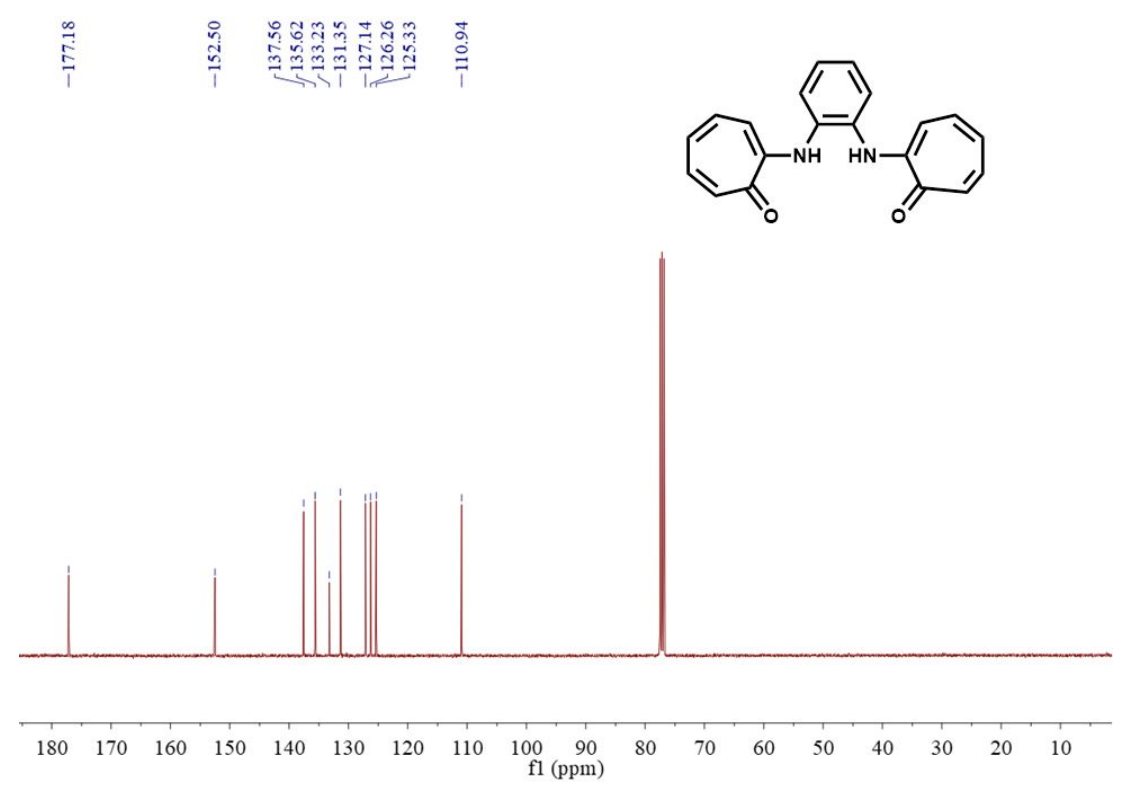

Figure S2. ${ }^{13} \mathrm{C}$ NMR spectrum of 2,2'-(1,2-phenylenebis(azan-ediyl))bis(cyclohepta-2,4,6-trien-1-one) in $\mathrm{CDCl}_{3}$.

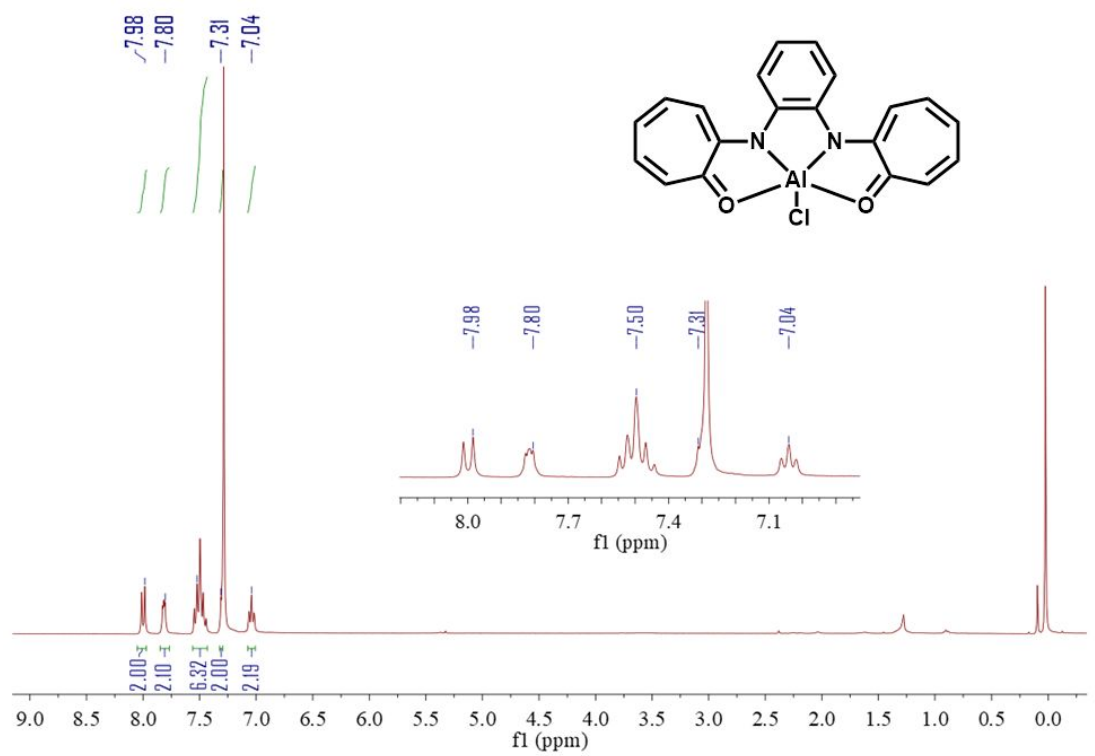

Figure S3. ${ }^{1} \mathrm{H}$ NMR spectrum of $\mathrm{Al}^{\mathrm{III}}$ (tralen) $\mathrm{Cl}$ in $\mathrm{CDCl}_{3}$. 


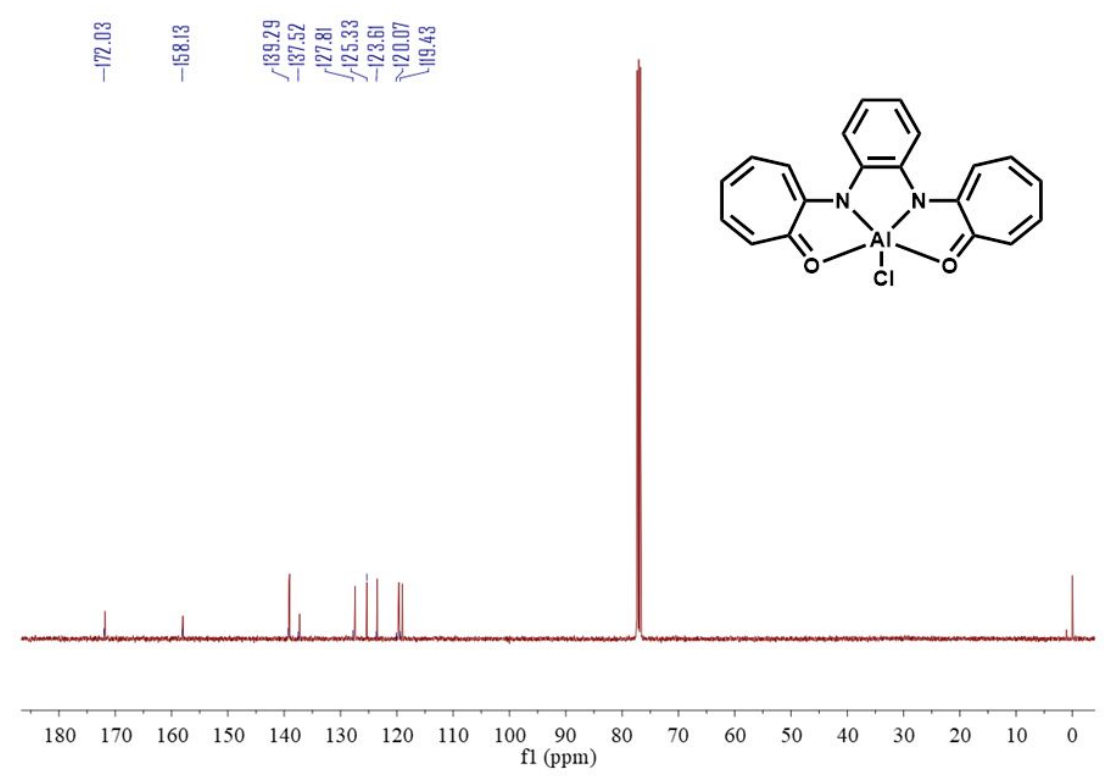

Figure S4. ${ }^{13} \mathrm{C}$ NMR spectrum of $\mathrm{Al}^{\mathrm{III}}$ (tralen) $\mathrm{Cl}$ in $\mathrm{CDCl}_{3}$.

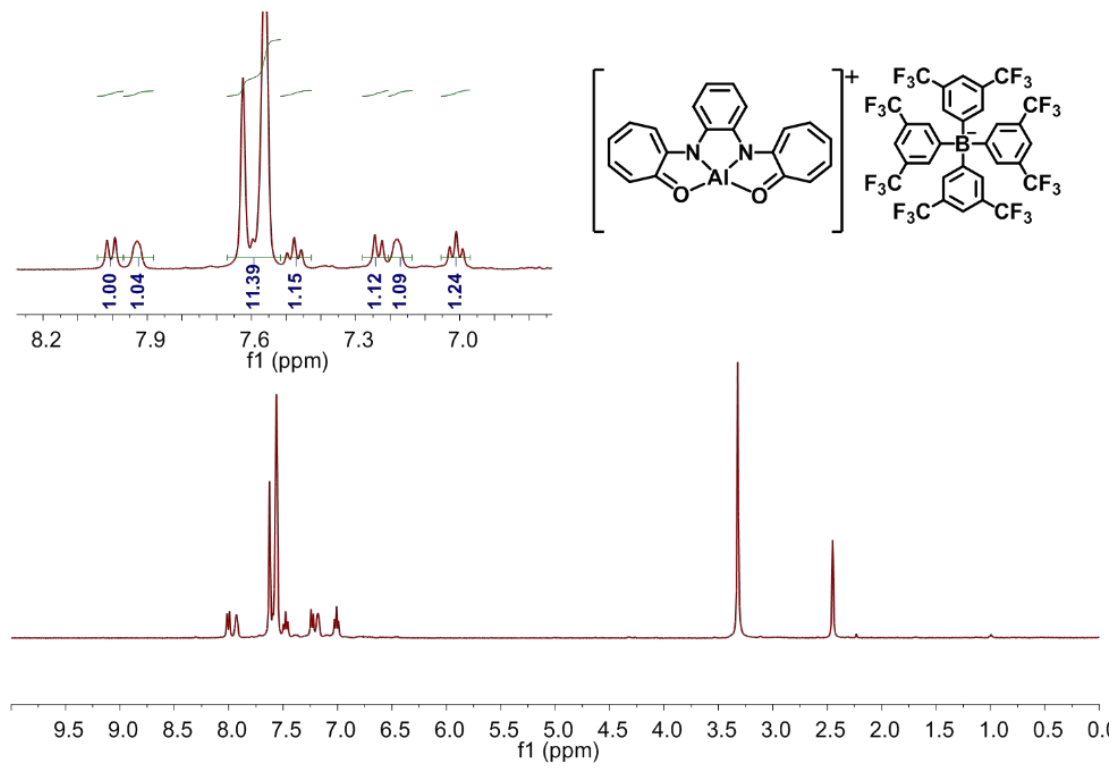

Figure S5. ${ }^{1} \mathrm{H}$ NMR spectrum of $\mathrm{Al}^{\mathrm{IIII}}$ (tralen)BArF in DMSO- $d_{6}$. 


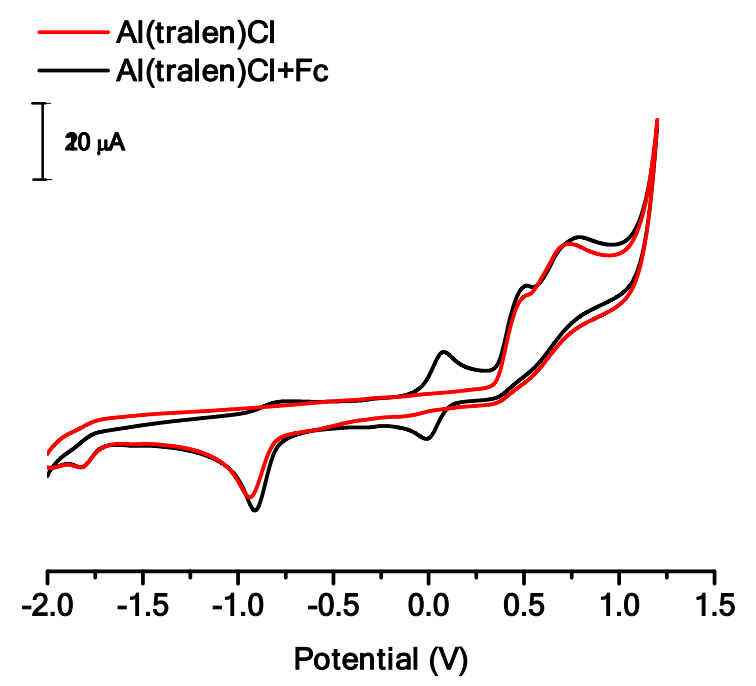

Figure S6. Cyclic voltammogram of $\mathrm{Al}($ tralen$) \mathrm{Cl}$ measured in $\mathrm{DMSO}$ at room temperature $\left(2 \mathrm{mM}\right.$ complex, $0.1 \mathrm{M} \mathrm{TBAPF}_{6}$, the scan rate was $\left.0.1 \mathrm{~V} \mathrm{~s}^{-1}\right)$. The potentials were referred to the ferrocene/ferrocenium $(\mathrm{Fc} / \mathrm{Fc}+)$ couple.

\section{The ORTEP diagram of $\mathrm{AI}^{\mathrm{III}}($ tralen)Cl}

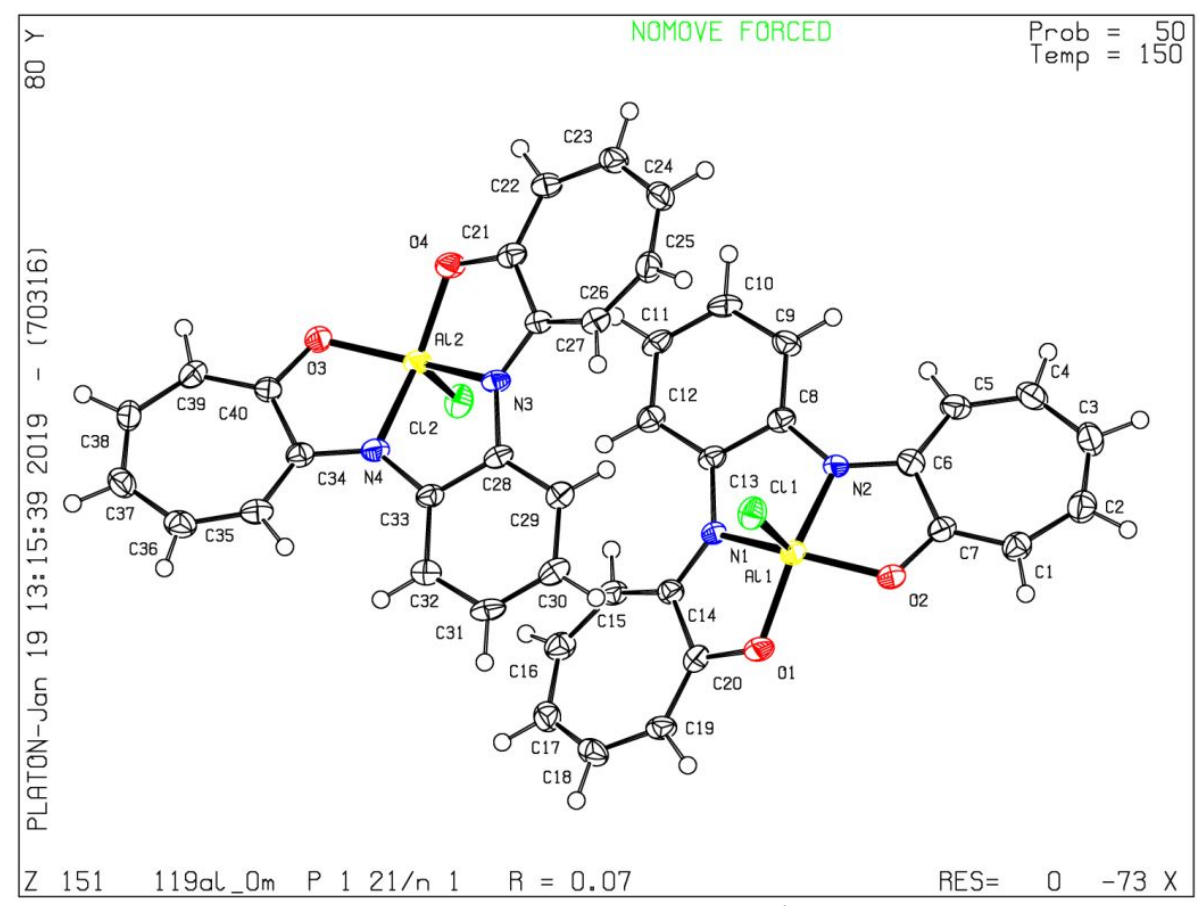

Figure S7. The ORTEP diagram of $\mathrm{Al}^{\mathrm{III}}$ (tralen)Cl. Selected bond lengths $(\AA)$ and angles $\left({ }^{\circ}\right)$ : $\mathrm{Cl}(1)-\mathrm{Al}(1) 2.1803(15), \mathrm{Al}(1)-$ $\mathrm{O}(1)$ 1.820(3), $\mathrm{Al}(1) 1-\mathrm{O}(2)$ 1.810(3), $\mathrm{Al}(1)-\mathrm{N}(1)$ 1.918(3), $\mathrm{Al}(1)-\mathrm{N}(2)$ 1.933(3), O(1)-Al(1)-N(1) 82.73(12), O(1)-Al(1)-N(2) 147.10(13), O(2)-Al(1)-O(1) 96.35(12), O(2)-Al(1)-N(1) 147.05(13), O(2)-Al(1)-N(2) 82.80(12), N(1)-Al(1)-N(2) 80.74(13). 
Experimental data of each polymerization

Table S1. VAc polymerization mediated by $\mathrm{Al}^{\mathrm{III}}$ (tralen)Cl in bulk under the condition of $\left[\mathrm{Al}{ }^{\mathrm{III}} \text { (tralen) } \mathrm{Cl}\right]_{0} /[\mathrm{NaBArF}]_{0} /[\mathrm{AIBN}]_{0} /[\mathrm{VAc}]_{0}=1 / 0 / 20 / 1000$ at $60{ }^{\circ} \mathrm{C}$.

\begin{tabular}{ccccc}
\hline $\begin{array}{c}\text { Time } \\
(\mathrm{min})\end{array}$ & $\begin{array}{c}\text { Conv. }^{\mathrm{a}} \\
(\%)\end{array}$ & $\begin{array}{c}M_{\mathrm{n}, \exp }{ }^{\mathrm{b}} \\
(\mathrm{g} / \mathrm{mol})\end{array}$ & $\begin{array}{c}M_{\mathrm{n}, \mathrm{h}^{\mathrm{c}}} \\
(\mathrm{g} / \mathrm{mol})\end{array}$ & PDI $^{\mathrm{b}}$ \\
\hline 60 & 0 & - & - & - \\
120 & 4 & 14,700 & 3,300 & 1.51 \\
180 & 5 & 16,200 & 4,300 & 1.48 \\
240 & 10 & 19,300 & 9,000 & 1.62 \\
300 & 22 & 27,700 & 19,100 & 1.78 \\
360 & 45 & 42,600 & 39,100 & 2.06 \\
\hline
\end{tabular}

${ }^{\mathrm{a} C}$ Conversion was detected by ${ }^{1} \mathrm{H}$ NMR. ${ }^{\mathrm{b}} M_{\mathrm{n}}$ and PDI were determined by gel permeation chromatography (GPC) in THF with polystyrene as standard. ${ }^{\mathrm{c}} M_{\mathrm{n}, \mathrm{th}}=M_{\mathrm{w}(\text { mediator })}+M_{\mathrm{w}(\mathrm{VAc})} \times\left([\mathrm{VAc}]_{0} /\left[\mathrm{Al}{ }^{\mathrm{III}}\right]_{0}\right) \times$ conv..

Table S2. VAc polymerization mediated by $\mathrm{Al}^{\mathrm{III}}($ tralen $) \mathrm{Cl}$ in bulk under the condition of $\left[\mathrm{Al} 1^{\mathrm{III}} \text { (tralen) } \mathrm{Cl}\right]_{0} /[\mathrm{NaBArF}]_{0} /[\mathrm{AIBN}]_{0} /[\mathrm{VAc}]_{0}=1 / 1 / 20 / 1000$ at $60{ }^{\circ} \mathrm{C}$.

\begin{tabular}{ccccc}
\hline $\begin{array}{c}\text { Time } \\
(\mathrm{min})\end{array}$ & $\begin{array}{c}\text { Conv. } \\
(\%)\end{array}$ & $\begin{array}{c}M_{\mathrm{n}, \text { exp }}^{\mathrm{b}} \\
(\mathrm{g} / \mathrm{mol})\end{array}$ & $\begin{array}{c}M_{\mathrm{n}, \mathrm{th}}^{\mathrm{c}} \\
(\mathrm{g} / \mathrm{mol})\end{array}$ & PDI $^{\mathrm{b}}$ \\
\hline 60 & 0 & - & - & - \\
150 & 0 & - & - & - \\
240 & 7 & 9,400 & 6,000 & 1.94 \\
330 & 13 & 14,900 & 11,100 & 2.02 \\
420 & 21 & 22,300 & 18,100 & 1.96 \\
510 & 38 & 37,900 & 32,700 & 2.03 \\
630 & 48 & 45,300 & 41,300 & 2.17 \\
\hline
\end{tabular}

${ }^{a}$ Conversion was detected by ${ }^{1} \mathrm{H}$ NMR. ${ }^{\mathrm{b}} M_{\mathrm{n}}$ and PDI were determined by gel permeation chromatography (GPC) in THF with polystyrene as standard. ${ }^{\mathrm{c}} M_{\mathrm{n}, \mathrm{th}}=M_{\mathrm{w}(\text { mediator })}+M_{\mathrm{w}(\mathrm{VAc})} \times\left([\mathrm{VAc}]_{0} /\left[\mathrm{Al}{ }^{\mathrm{II}}\right]_{0}\right) \times$ conv..

Table S3. VAc polymerization mediated by $\mathrm{Al}^{\mathrm{III}}(\mathrm{tralen}) \mathrm{Cl}$ in bulk under the condition of $\left[\mathrm{Al}{ }^{\mathrm{III}} \text { (tralen) } \mathrm{Cl}\right]_{0} /[\mathrm{NaBArF}]_{0} /[\mathrm{AIBN}]_{0} /[\mathrm{VAc}]_{0}=1 / 3 / 20 / 1000$ at $60^{\circ} \mathrm{C}$.

\begin{tabular}{ccccc}
\hline $\begin{array}{c}\text { Time } \\
(\mathrm{min})\end{array}$ & $\begin{array}{c}\text { Conv. }^{\mathrm{a}} \\
(\%)\end{array}$ & $\begin{array}{c}M_{\mathrm{n}, \mathrm{exp}}{ }^{\mathrm{b}} \\
(\mathrm{g} / \mathrm{mol})\end{array}$ & $\begin{array}{c}M_{\mathrm{n}, \mathrm{th}}{ }^{\mathrm{c}} \\
(\mathrm{g} / \mathrm{mol})\end{array}$ & PDI $^{\mathrm{b}}$ \\
\hline 90 & 0 & - & - & - \\
180 & 0 & - & - & - \\
240 & 6 & 8,000 & 5,000 & 1.74 \\
330 & 11 & 11,300 & 9,400 & 2.08 \\
42 & 16 & 16,300 & 13,700 & 2.07 \\
510 & 26 & 27,400 & 22,300 & 1.93 \\
660 & 42 & 38,100 & 36,100 & 2.10 \\
\hline
\end{tabular}


${ }^{\mathrm{a} C}$ Conversion was detected by ${ }^{1} \mathrm{H}$ NMR. ${ }^{\mathrm{b}} M_{\mathrm{n}}$ and PDI were determined by gel permeation chromatography (GPC) in THF with polystyrene as standard. ${ }^{\mathrm{c}} M_{\mathrm{n}, \mathrm{th}}=M_{\mathrm{w}(\text { mediator })}+M_{\mathrm{w}(\mathrm{VAc})} \times\left([\mathrm{VAc}]_{0} /\left[\mathrm{Al}^{\mathrm{III}}\right]_{0}\right) \times$ conv..
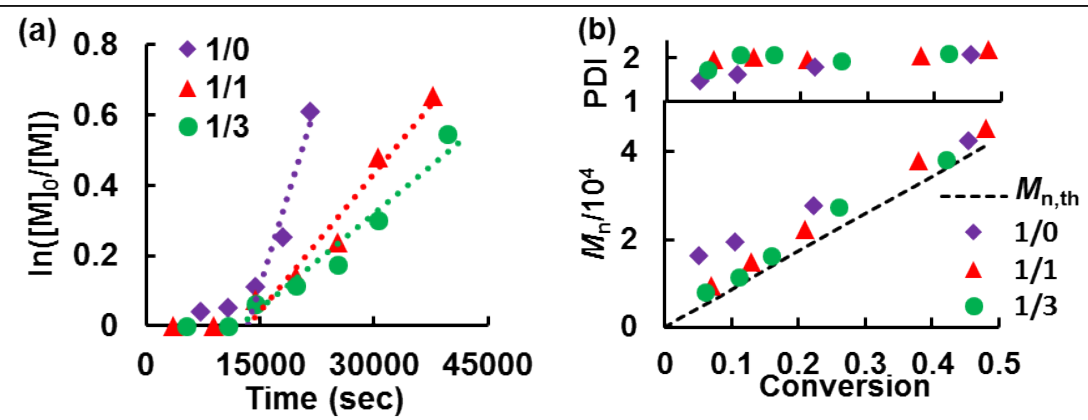

Figure S8. VAc polymerization mediated by $\mathrm{Al}{ }^{\mathrm{III}}(\operatorname{tralen}) \mathrm{Cl}$ at $60{ }^{\circ} \mathrm{C}$ in bulk under the condition of $\left[\mathrm{Al}{ }^{\mathrm{III}} \text { (tralen) } \mathrm{Cl}\right]_{0} /[\mathrm{NaBArF}]_{0} /[\mathrm{AIBN}]_{0} /[\mathrm{VAc}]_{0}=1 / \mathrm{x} / 20 / 1000$ with $[\mathrm{VAc}]_{0}=10.84 \mathrm{M}$. (a) First-order kinetic plots. The slopes in the kinetic plots are $6.89 \times 10^{-5} \mathrm{~s}^{-1}(\diamond, \mathrm{x}=0), 2.62 \times 10^{-5} \mathrm{~s}^{-1}(\boldsymbol{\Lambda}, \mathrm{x}=1)$, and $1.81 \times 10^{-5} \mathrm{~s}^{-1}(\bullet, \mathrm{x}=3)$. (b) The plots of $M_{\mathrm{n}}$ and PDI versus conversion, $(\bullet) \mathrm{x}=0,(\boldsymbol{\Delta}) \mathrm{x}=1$, and $(\bullet) \mathrm{x}=3$.
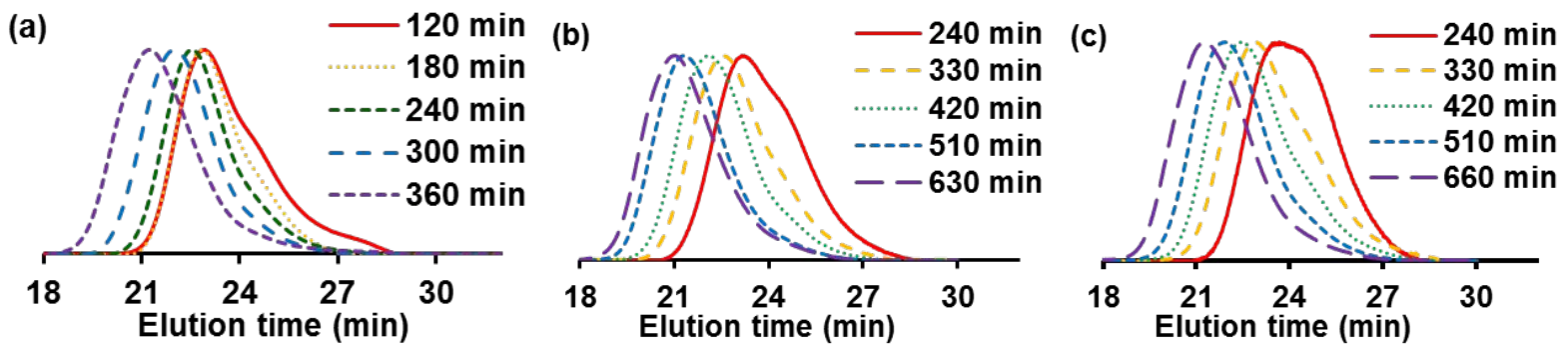

Figure S9. The GPC traces for VAc polymerization mediated by $\mathrm{Al}$ III (tralen) $\mathrm{Cl}$ at $60{ }^{\circ} \mathrm{C}$ in bulk under the following condition of $\left[\mathrm{Al}^{\mathrm{III}} \text { (tralen) } \mathrm{Cl}\right]_{0} /[\mathrm{NaBArF}]_{0} /[\mathrm{AIBN}]_{0} /[\mathrm{VAc}]_{0}=1 / \mathrm{X} / 20 / 1000$, (a) $\mathrm{X}=0$, (b) $\mathrm{X}=1$ and (c) $\mathrm{X}=3$.

Table S4. VAc polymerization mediated by $\mathrm{Al}^{\mathrm{III}}(\operatorname{tralen}) \mathrm{Cl}$ in bulk under the condition of $\left[\mathrm{Al}^{\mathrm{III}} \text { (tralen) } \mathrm{Cl}\right]_{0} /[\mathrm{NaBArF}]_{0} /[\mathrm{AIBN}]_{0} /[\mathrm{VAc}]_{0}=1 / 1 / 10 / 1000$ at $60{ }^{\circ} \mathrm{C}$.

\begin{tabular}{ccccc}
\hline $\begin{array}{c}\text { Time } \\
(\mathrm{min})\end{array}$ & $\begin{array}{c}\text { Conv. }^{\mathrm{a}} \\
(\%)\end{array}$ & $\begin{array}{c}M_{\mathrm{n}, \exp }{ }^{\mathrm{b}} \\
(\mathrm{g} / \mathrm{mol})\end{array}$ & $\begin{array}{c}M_{\mathrm{n}, \mathrm{th}^{\mathrm{c}}} \\
(\mathrm{g} / \mathrm{mol})\end{array}$ & PDI $^{\mathrm{b}}$ \\
\hline 180 & 0 & - & - & - \\
300 & 0 & - & - & - \\
420 & 4 & 3,800 & 3,400 & 1.69 \\
540 & 12 & 12,900 & 10,300 & 1.56 \\
660 & 17 & 16,300 & 14,600 & 2.15 \\
780 & 25 & 23,000 & 21,500 & 2.37 \\
900 & 32 & 29,100 & 27,500 & 2.21 \\
\hline
\end{tabular}

${ }^{\mathrm{a} C}$ Conversion was detected by ${ }^{1} \mathrm{H}$ NMR. ${ }^{\mathrm{b}} M_{\mathrm{n}}$ and PDI were determined by gel permeation chromatography (GPC) in THF with polystyrene as standard. ${ }^{\mathrm{c}} M_{\mathrm{n}, \mathrm{th}}=M_{\mathrm{w}(\text { mediator })}+M_{\mathrm{w}(\mathrm{VAc})} \times\left([\mathrm{VAc}]_{0} /\left[\mathrm{Al}^{\mathrm{III}}\right]_{0}\right) \times$ conv.. 
Table S5. VAc polymerization mediated by $\mathrm{Al}^{\mathrm{III}}(\operatorname{tralen}) \mathrm{Cl}$ in bulk under the condition of $\left[\mathrm{Al}{ }^{\mathrm{III}} \text { (tralen) } \mathrm{Cl}\right]_{0} /[\mathrm{NaBArF}]_{0} /[\mathrm{AIBN}]_{0} /[\mathrm{VAc}]_{0}=1 / 1 / 40 / 1000$ at $60^{\circ} \mathrm{C}$.

\begin{tabular}{ccccc}
\hline $\begin{array}{c}\text { Time } \\
(\mathrm{min})\end{array}$ & $\begin{array}{c}\text { Conv. }^{\mathrm{a}} \\
(\%)\end{array}$ & $\begin{array}{c}M_{\mathrm{n}, \exp }{ }^{\mathrm{b}} \\
(\mathrm{g} / \mathrm{mol})\end{array}$ & $\begin{array}{c}M_{\mathrm{n}, \mathrm{th}^{\mathrm{c}}} \\
(\mathrm{g} / \mathrm{mol})\end{array}$ & PDI $^{\mathrm{b}}$ \\
\hline 120 & 0 & - & - & - \\
150 & 0 & - & - & - \\
180 & 5 & 10,800 & 4,400 & 1.46 \\
210 & 7 & 12,400 & 6,800 & 1.76 \\
240 & 13 & 16,200 & 11,200 & 1.75 \\
270 & 19 & 21,800 & 16,500 & 1.79 \\
300 & 28 & 25,000 & 24,600 & 2.03 \\
330 & 39 & 35,500 & 34,200 & 1.94 \\
360 & 48 & 38,600 & 41,200 & 2.19 \\
\hline
\end{tabular}

${ }^{\mathrm{a} C o n v e r s i o n}$ was detected by ${ }^{1} \mathrm{H}$ NMR. ${ }^{\mathrm{b}} M_{\mathrm{n}}$ and PDI were determined by gel permeation chromatography (GPC) in THF with polystyrene as standard. ${ }^{\mathrm{c}} M_{\mathrm{n}, \mathrm{th}}=M_{\mathrm{w}(\text { mediator })}+M_{\mathrm{w}(\mathrm{VAc})} \times\left([\mathrm{VAc}]_{0} /\left[\mathrm{Al}{ }^{\mathrm{III}}\right]_{0}\right) \times$ conv..
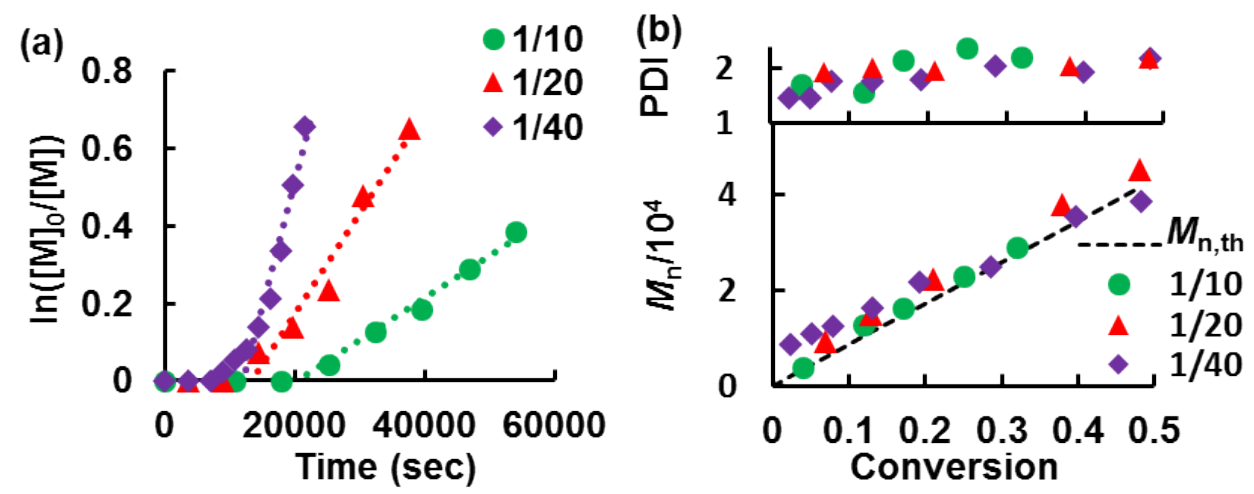

Figure S10. VAc polymerization mediated by $\mathrm{Al}^{\mathrm{III}}(\operatorname{tralen}) \mathrm{Cl}$ at $60{ }^{\circ} \mathrm{C}$ in bulk under the condition of $\left[\mathrm{Al}{ }^{\mathrm{III}} \text { (tralen)Cl }\right]_{0} /[\mathrm{NaBArF}]_{0} /[\mathrm{AIBN}]_{0} /[\mathrm{VAc}]_{0}=1 / 1 / \mathrm{x} / 1000$ with $[\mathrm{VAc}]_{0}=10.84 \mathrm{M}$. (a) First-order kinetic plots. The slopes in the kinetic plots are $1.08 \times 10^{-5} \mathrm{~s}^{-1}(\mathbf{x}=10), 2.62 \times 10^{-5} \mathrm{~s}^{-1}(\boldsymbol{\Lambda}, \mathrm{x}=20)$, and $6.51 \times 10^{-5} \mathrm{~s}^{-1}(\diamond, \mathrm{x}=40)$. (b) The plots of $M_{\mathrm{n}}$ and PDI versus conversion, $(\mathbf{O}) \mathrm{x}=10,(\boldsymbol{\Delta}) \mathrm{x}=20$, and $(\diamond) \mathrm{x}=40$. 

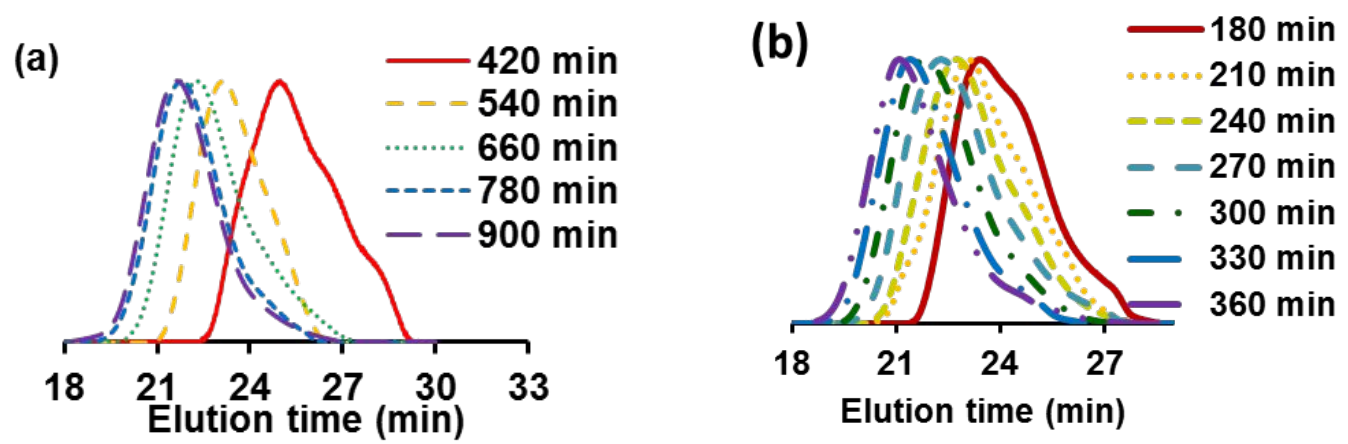

Figure S11. The GPC traces for VAc polymerization mediated by $\mathrm{Al}{ }^{\mathrm{III}}\left(\right.$ tralen) $\mathrm{Cl}$ at $60^{\circ} \mathrm{C}$ in bulk under the following condition of $\left[\mathrm{Al}{ }^{\mathrm{III}}(\text { tralen }) \mathrm{Cl}\right]_{0} /[\mathrm{NaBArF}]_{0} /[\mathrm{AIBN}]_{0} /[\mathrm{VAc}]_{0}=1 / 1 / \mathrm{X} / 1000$, (a) $\mathrm{X}=10$ and (b) $\mathrm{X}=40$.

Table S6. VAc polymerization mediated by $\mathrm{Al}^{\mathrm{III}}(\mathrm{tralen}) \mathrm{Cl}$ in bulk under the condition of $\left[\mathrm{Al}{ }^{\mathrm{III}} \text { (tralen) } \mathrm{Cl}\right]_{0} /[\mathrm{NaBArF}]_{0} /[\mathrm{AIBN}]_{0} /[\mathrm{VAc}]_{0}=1 / 1 / 20 / 500$ at $60{ }^{\circ} \mathrm{C}$.

\begin{tabular}{ccccc}
\hline $\begin{array}{c}\text { Time } \\
(\mathrm{min})\end{array}$ & $\begin{array}{c}\text { Conv }^{\mathrm{a}} \\
(\%)\end{array}$ & $\begin{array}{c}M_{\mathrm{n}, \exp }{ }^{\mathrm{b}} \\
(\mathrm{g} / \mathrm{mol})\end{array}$ & $\begin{array}{c}M_{\mathrm{n}, \mathrm{th}^{\mathrm{c}}} \\
(\mathrm{g} / \mathrm{mol})\end{array}$ & PDI $^{\mathrm{b}}$ \\
\hline 60 & 0 & - & - & - \\
180 & 3 & 3,000 & 1,300 & 1.57 \\
300 & 12 & 8,700 & 5,100 & 2.12 \\
420 & 25 & 13,200 & 10,700 & 2.38 \\
540 & 43 & 24,400 & 18,500 & 2.37 \\
660 & 66 & 36,600 & 28,400 & 1.48 \\
\hline
\end{tabular}

${ }^{\mathrm{a} C}$ Conversion was detected by ${ }^{1} \mathrm{H}$ NMR. ${ }^{\mathrm{b}} M_{\mathrm{n}}$ and PDI were determined by gel permeation chromatography (GPC) in THF with polystyrene as standard. ${ }^{\mathrm{c}} M_{\mathrm{n}, \mathrm{th}}=M_{\mathrm{w}(\text { mediator })}+M_{\mathrm{w}(\mathrm{VAc})} \times\left([\mathrm{VAc}]_{0} /[\mathrm{Al}]_{0}\right) \times$ conv..

Table S7. VAc polymerization mediated by $\mathrm{Al}^{\mathrm{III}}($ tralen)Cl in bulk under the condition of $\left[\mathrm{Al}{ }^{\mathrm{III}} \text { (tralen) } \mathrm{Cl}\right]_{0} /[\mathrm{NaBArF}]_{0} /[\mathrm{AIBN}]_{0} /[\mathrm{VAc}]_{0}=1 / 1 / 20 / 4000$ at $60{ }^{\circ} \mathrm{C}$.

\begin{tabular}{ccccc}
\hline $\begin{array}{c}\text { Time } \\
(\mathrm{min})\end{array}$ & $\begin{array}{c}\text { Conv. }^{\mathrm{a}} \\
(\%)\end{array}$ & $\begin{array}{c}M_{\mathrm{n}, \mathrm{exp}}{ }^{\mathrm{b}} \\
(\mathrm{g} / \mathrm{mol})\end{array}$ & $\begin{array}{c}M_{\mathrm{n}, \mathrm{th}^{\mathrm{c}}} \\
(\mathrm{g} / \mathrm{mol})\end{array}$ & PDI $^{\mathrm{b}}$ \\
\hline 60 & 0 & - & - & - \\
120 & 0 & - & - & - \\
180 & 0 & - & - & - \\
240 & 4 & 14,800 & 13,700 & 1.86 \\
330 & 10 & 42,300 & 34,400 & 1.77 \\
450 & 18 & 60,500 & 61,900 & 1.97 \\
570 & 26 & 95,900 & 89,500 & 1.92 \\
720 & 35 & 111,800 & 120,500 & 2.08 \\
\hline
\end{tabular}

${ }^{\mathrm{a} C o n v e r s i o n ~ w a s ~ d e t e c t e d ~ b y ~}{ }^{1} \mathrm{H}$ NMR. ${ }^{\mathrm{b}} M_{\mathrm{n}}$ and PDI were determined by gel permeation chromatography (GPC) in THF with polystyrene as standard. ${ }^{\mathrm{c}} M_{\mathrm{n}, \mathrm{th}}=M_{\mathrm{w}(\text { mediator })}+M_{\mathrm{w}(\mathrm{VAc})} \times\left([\mathrm{VAc}]_{0} /\left[\mathrm{Al}^{\mathrm{III}}\right]_{0}\right) \times$ conv.. 

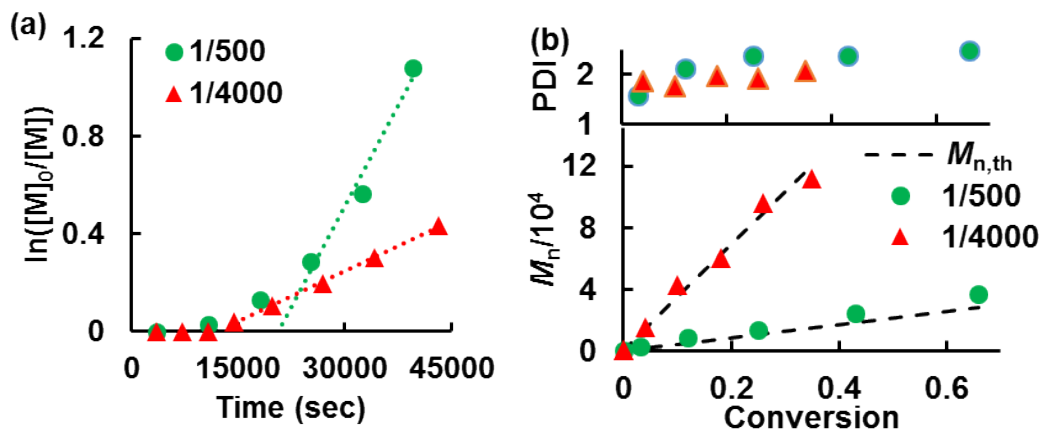

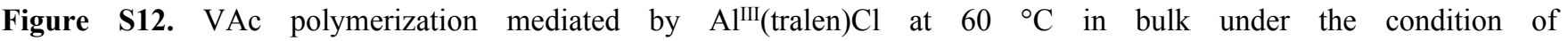
$\left[\mathrm{Al}{ }^{\mathrm{III}} \text { (tralen) } \mathrm{Cl}\right]_{0} /[\mathrm{NaBArF}]_{0} /[\mathrm{AIBN}]_{0} /[\mathrm{VAc}]_{0}=1 / 1 / 20 / \mathrm{x}$ with $[\mathrm{VAc}]_{0}=10.84 \mathrm{M}$. (a) First-order kinetic plots. The slopes in the kinetic plots are $5.49 \times 10^{-5} \mathrm{~s}^{-1}(\mathbf{x}=500)$, and $1.36 \times 10^{-5} \mathrm{~s}^{-1}(\boldsymbol{\Lambda}, \mathrm{x}=4000)$. (b) The plots of $M_{\mathrm{n}}$ and PDI versus conversion, $\mathrm{x}=500$, and $(\mathbf{\Delta}) \mathrm{x}=4000$.
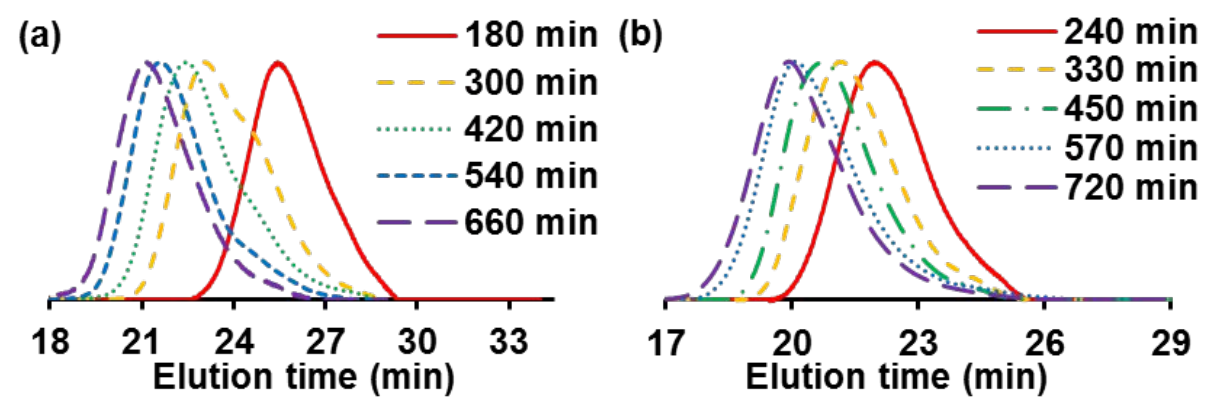

Figure S13. The GPC traces for VAc polymerization mediated by $\mathrm{Al}{ }^{\mathrm{III}}($ tralen$) \mathrm{Cl}$ at $60^{\circ} \mathrm{C}$ in bulk under the following condition of $\left[\mathrm{Al}{ }^{\mathrm{III}}(\operatorname{tralen}) \mathrm{Cl}\right]_{0} /[\mathrm{NaBArF}]_{0} /[\mathrm{AIBN}]_{0} /[\mathrm{VAc}]_{0}=1 / 1 / 20 / \mathrm{X}$, (a) $\mathrm{X}=500$ and (b) $\mathrm{X}=4000$.

Table S8. VAc polymerization mediated by $\mathrm{Al}^{\mathrm{IIII}}(\operatorname{tralen}) \mathrm{Cl}$ in bulk under the condition of $\left[\mathrm{Al}{ }^{\mathrm{III}} \text { (tralen) } \mathrm{Cl}\right]_{0} /[\mathrm{NaBArF}]_{0} /[\mathrm{AIBN}]_{0} /[\mathrm{VAc}]_{0}=1 / 1 / 5 / 1000$ at $60^{\circ} \mathrm{C}$.

\begin{tabular}{ccccc}
\hline $\begin{array}{c}\text { Time } \\
(\mathrm{h})\end{array}$ & $\begin{array}{c}\text { Conv. }^{\mathrm{a}} \\
(\%)\end{array}$ & $\begin{array}{c}M_{\mathrm{n}, \exp }{ }^{\mathrm{b}} \\
(\mathrm{g} / \mathrm{mol})\end{array}$ & $\begin{array}{c}M_{\mathrm{n}, \mathrm{th}^{\mathrm{c}}} \\
(\mathrm{g} / \mathrm{mol})\end{array}$ & PDI $^{\mathrm{b}}$ \\
\hline 6 & 0 & - & - & - \\
12 & 0 & - & - & - \\
18 & 0 & - & - & - \\
24 & 0 & - & - & - \\
30 & 1 & 3,200 & 1000 & 1.75 \\
48 & 5 & 4,000 & 4300 & 1.93 \\
60 & 7 & 4,000 & 5100 & 1.87 \\
\hline
\end{tabular}

${ }^{a}$ Conversion was detected by ${ }^{1} \mathrm{H}$ NMR. ${ }^{\mathrm{b}} M_{\mathrm{n}}$ and PDI were determined by gel permeation chromatography (GPC) in THF with polystyrene as standard. ${ }^{\mathrm{c}} M_{\mathrm{n}, \mathrm{th}}=M_{\mathrm{w}(\text { mediator })}+M_{\mathrm{w}(\mathrm{VAc})} \times\left([\mathrm{VAc}]_{0} /\left[\mathrm{Al}{ }^{\mathrm{III}}\right]_{0}\right) \times$ conv.. 


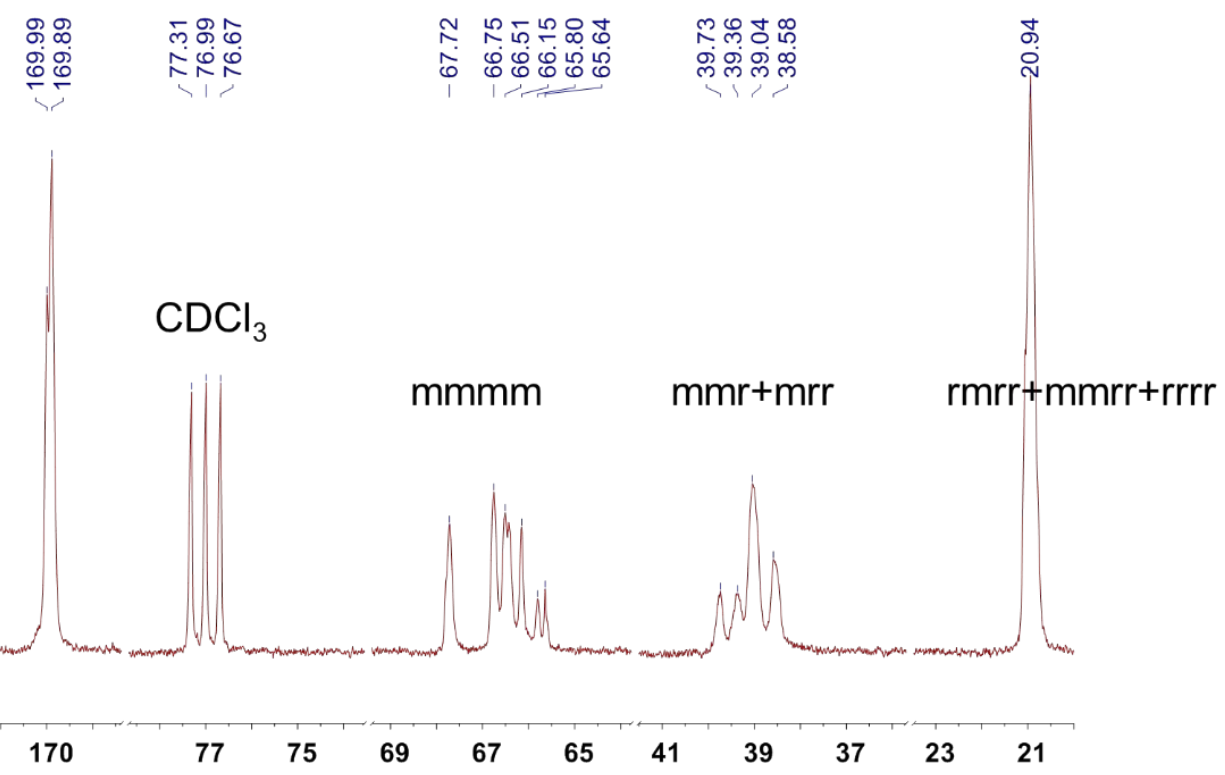

Figure S14. ${ }^{13} \mathrm{C}$ NMR spectrum of PVAc

Table S9. NVP polymerization mediated by $\mathrm{Al}^{\mathrm{III}}($ tralen$) \mathrm{Cl}$ in $\mathrm{DMF}$ under the condition of $\left[\mathrm{Al}{ }^{\mathrm{III}} \text { (tralen) } \mathrm{Cl}\right]_{0} /[\mathrm{NaBArF}]_{0} /[\mathrm{AIBN}]_{0} /[N \mathrm{VP}]_{0}=1 / 1 / 20 / 500$ at $60{ }^{\circ} \mathrm{C},[N \mathrm{VP}]_{0}=4.67 \mathrm{M}$

\begin{tabular}{|c|c|c|c|c|}
\hline $\begin{array}{l}\text { Time } \\
(\min )\end{array}$ & $\begin{array}{c}\text { Conv. }{ }^{\mathrm{a}} \\
(\%)\end{array}$ & $\begin{array}{l}M_{\mathrm{n}, \exp }{ }^{\mathrm{b}} \\
(\mathrm{g} / \mathrm{mol})\end{array}$ & $\begin{array}{c}M_{\mathrm{n}, \mathrm{th}}^{\mathrm{c}} \\
(\mathrm{g} / \mathrm{mol})\end{array}$ & $\mathrm{PDI}^{\mathrm{b}}$ \\
\hline 60 & 0 & - & - & - \\
\hline 120 & 7 & 8,400 & 4,100 & 1.69 \\
\hline 150 & 22 & 14,300 & 12,700 & 1.86 \\
\hline 180 & 39 & 21,000 & 21,900 & 2.00 \\
\hline 210 & 52 & 27,600 & 29,200 & 1.85 \\
\hline 240 & 68 & 27,500 & 37,800 & 2.08 \\
\hline 270 & 76 & 28,700 & 42,300 & 2.13 \\
\hline
\end{tabular}

${ }^{a}$ Conversion was detected by ${ }^{1} \mathrm{H}$ NMR. ${ }^{\mathrm{b}} M_{\mathrm{n}}$ and PDI were determined by gel permeation chromatography (GPC) in DMF with poly(ethylene glycol) $(\mathrm{PEG})$ as standard corrected by the Mark-Houwink equation. ${ }^{\mathrm{c}} M_{\mathrm{n}, \mathrm{th}}=M_{\mathrm{w}(\mathrm{mediator})}+$ $M_{\mathrm{w}(N \mathrm{VP})} \times\left([N \mathrm{VP}]_{0} /\left[\mathrm{Al}^{\mathrm{III}}\right]_{0}\right) \times$ conv.. 
Table S10. MA polymerization mediated by $\mathrm{Al}^{\mathrm{III}}($ tralen) $\mathrm{Cl}$ in $\mathrm{DMF}$ under the condition of $\left[\mathrm{Al}^{\mathrm{III}} \text { (tralen) } \mathrm{Cl}\right]_{0} /[\mathrm{NaBArF}]_{0} /[\mathrm{AIBN}]_{0} /[\mathrm{MA}]_{0}=1 / 1 / 20 / 1000$ at $60^{\circ} \mathrm{C},[\mathrm{MA}]_{0}=5.53$.

\begin{tabular}{ccccc}
\hline Time (min) & $\begin{array}{c}\text { Conv. }^{\mathrm{a}} \\
(\%)\end{array}$ & $\begin{array}{c}M_{\mathrm{n}, \mathrm{exp}}^{\mathrm{b}} \\
\left(10^{3} \mathrm{~g} / \mathrm{mol}\right)\end{array}$ & $\begin{array}{c}M_{\mathrm{n}, \mathrm{th}^{\mathrm{c}}} \\
(\mathrm{g} / \mathrm{mol})\end{array}$ & PDI $^{\mathrm{b}}$ \\
\hline 5 & 0 & - & - & - \\
10 & 5 & 70,000 & 4,900 & 1.48 \\
15 & 15 & 65,400 & 13,400 & 1.56 \\
20 & 34 & 61,100 & 29,500 & 1.64 \\
25 & 43 & 60,200 & 37,900 & 1.70 \\
30 & 54 & 56,600 & 47,100 & 1.77 \\
35 & 57 & 70,100 & 50,000 & 1.61 \\
40 & 62 & 67,600 & 54,400 & 1.57 \\
\hline
\end{tabular}

${ }^{\mathrm{a} C}$ Conversion was detected by ${ }^{1} \mathrm{H}$ NMR. ${ }^{\mathrm{b}} M_{\mathrm{n}}$ and PDI were determined by gel permeation chromatography (GPC) in THF with polystyrene as standard. $\left.{ }^{c} M_{\mathrm{n}, \mathrm{th}}=M_{\mathrm{w}(\text { mediator })}+M_{\mathrm{w}(\mathrm{MA})} \times\left([\mathrm{MA}]_{0} /[\mathrm{Al}]^{\mathrm{III}}\right]_{0}\right) \times$ conv..
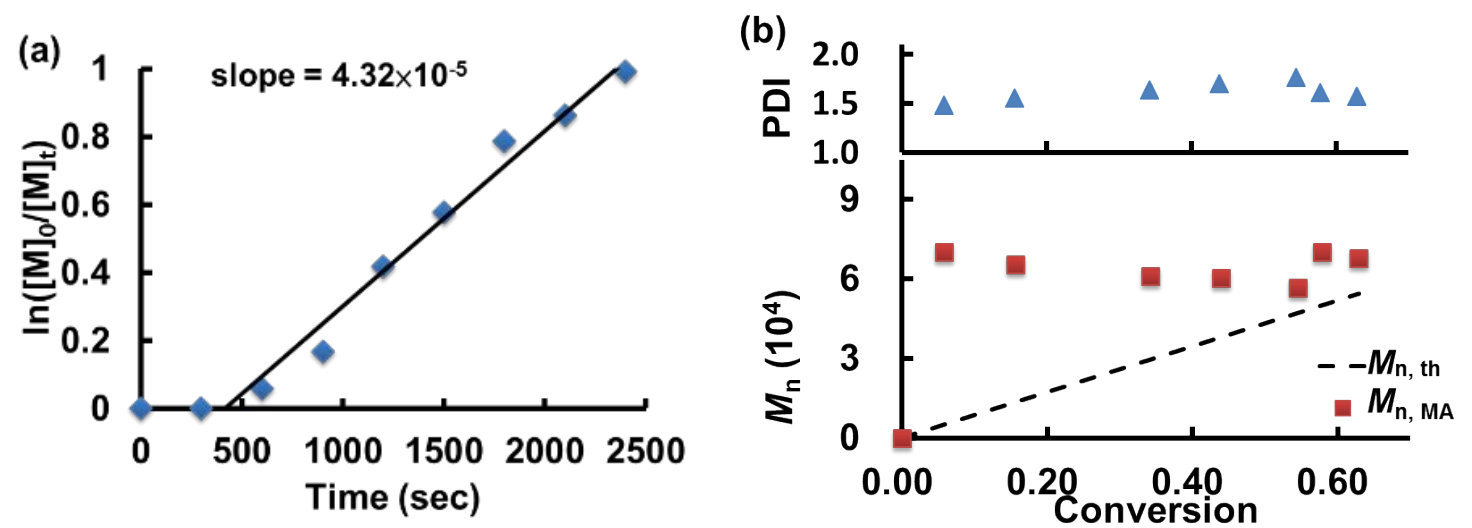

Figure S15. MA polymerization mediated by $\mathrm{Al}^{\mathrm{III}}($ tralen$) \mathrm{Cl}$ at $60{ }^{\circ} \mathrm{C}$ in $\mathrm{DMF}$ under the condition of $\left[\mathrm{Al}^{\mathrm{III}} \text { (tralen)Cl }\right]_{0} /[\mathrm{NaBArF}]_{0} /[\mathrm{AIBN}]_{0} /[\mathrm{MA}]_{0}=1 / 1 / 20 / 1000$ with $[\mathrm{MA}]_{0}=5.53 \mathrm{M}$. (a) First-order kinetic plots. (b) The plots of $M_{\mathrm{n}}$ and PDI versus conversion.

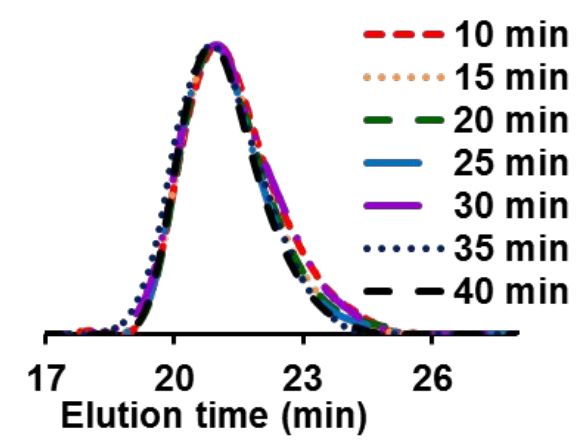

Figure S16. The GPC traces for MA polymerization mediated by $\mathrm{Al}^{\mathrm{III}}(\operatorname{tralen}) \mathrm{Cl}$ at $60^{\circ} \mathrm{C}$ in bulk under the following condition of $\left[\mathrm{Al}^{\mathrm{III}}(\operatorname{tralen}) \mathrm{Cl}\right]_{0} /[\mathrm{NaBArF}]_{0} /[\mathrm{AIBN}]_{0} /[\mathrm{MA}]_{0}=1 / 1 / 20 / 1000$. 
Table S11. AN polymerization mediated by $\mathrm{Al}^{\mathrm{III}}($ tralen $) \mathrm{Cl}$ in $\mathrm{DMF}$ under the condition of $\left[\mathrm{Al}^{\mathrm{III}}(\text { tralen}) \mathrm{Cl}\right]_{0} /[\mathrm{NaBArF}]_{0} /[\mathrm{AIBN}]_{0} /[\mathrm{AN}]_{0}=1 / 1 / 20 / 500$ at $60^{\circ} \mathrm{C},[\mathrm{AN}]_{0}=3.81 \mathrm{M}$.

\begin{tabular}{ccccc}
\hline $\begin{array}{c}\text { Time } \\
(\mathrm{min})\end{array}$ & $\begin{array}{c}\text { Conv. }^{\mathrm{a}} \\
(\%)\end{array}$ & $\begin{array}{c}M_{\mathrm{n}, \exp }{ }^{\mathrm{b}} \\
(\mathrm{g} / \mathrm{mol})\end{array}$ & $\begin{array}{c}M_{\mathrm{n}, \mathrm{th}}{ }^{\mathrm{c}} \\
(\mathrm{g} / \mathrm{mol})\end{array}$ & PDI $^{\mathrm{b}}$ \\
\hline 30 & 24 & 45,600 & 6,500 & 1.39 \\
60 & 55 & 47,500 & 14,600 & 1.43 \\
90 & 61 & 46,800 & 16,400 & 1.46 \\
120 & 73 & 48,900 & 19,400 & 1.45 \\
150 & 82 & 44,000 & 21,900 & 1.57 \\
\hline
\end{tabular}

${ }^{\mathrm{a} C o n v e r s i o n}$ was detected by ${ }^{1} \mathrm{H}$ NMR. ${ }^{\mathrm{b}} M_{\mathrm{n}}$ and PDI were determined by gel permeation chromatography (GPC) in DMF with poly(ethylene glycol) (PEG) as standard corrected by the Mark-Houwink equation. ${ }^{\mathrm{c}} M_{\mathrm{n}, \mathrm{th}}=M_{\mathrm{w} \text { (mediator) }}+$ $M_{\mathrm{w}(\mathrm{AN})} \times\left([\mathrm{AN}]_{0} /\left[\mathrm{Al} \mathrm{II}^{\mathrm{II}}\right]_{0}\right) \times$ conv..
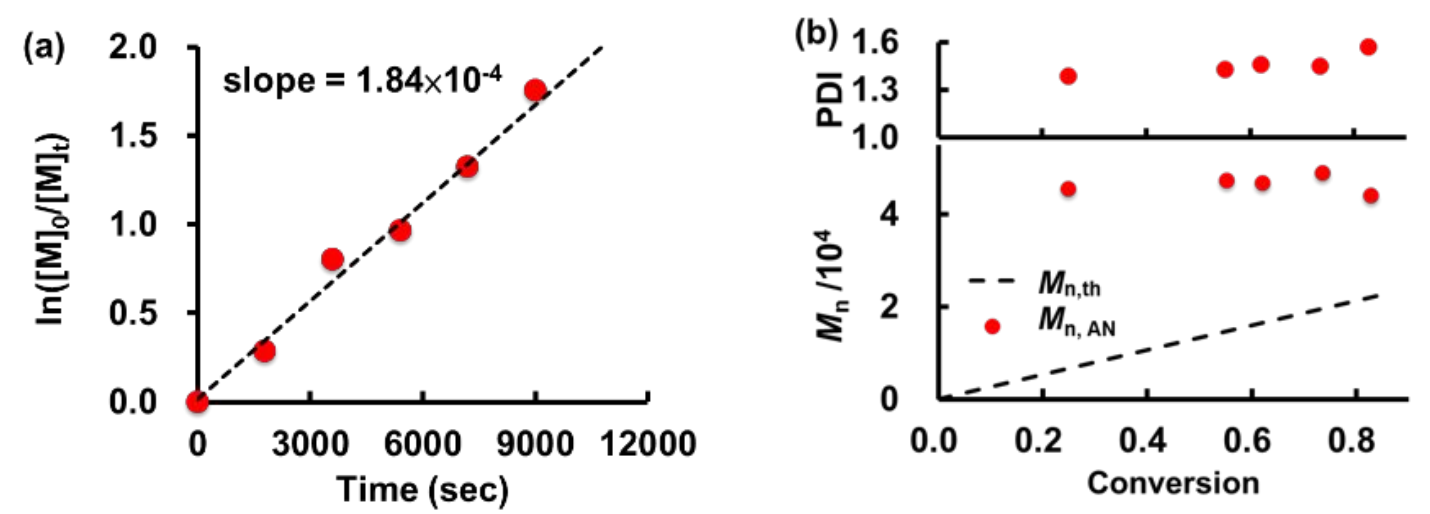

Figure S17. AN polymerization mediated by $\mathrm{Al}^{\mathrm{III}}(\operatorname{tralen}) \mathrm{Cl}$ at $60{ }^{\circ} \mathrm{C}$ in $\mathrm{DMF}$ under the condition of $\left[\mathrm{Al}^{\mathrm{III}} \text { (tralen) } \mathrm{Cl}\right]_{0} /[\mathrm{NaBArF}]_{0} /[\mathrm{AIBN}]_{0} /[\mathrm{AN}]_{0}=1 / 1 / 20 / 500$ with $[\mathrm{AN}]_{0}=3.81 \mathrm{M}$. (a) First-order kinetic plots. (b) The plots of $M_{\mathrm{n}}$ and PDI versus conversion.

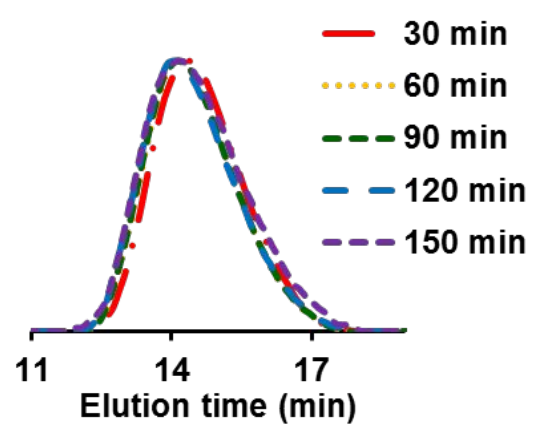

Figure S18. The GPC traces for AN polymerization mediated by $\mathrm{Al}{ }^{\mathrm{III}}$ (tralen) $\mathrm{Cl}$ at $60^{\circ} \mathrm{C}$ in bulk under the following condition of $\left[\mathrm{Al}{ }^{\mathrm{III}}(\text { tralen }) \mathrm{Cl}\right]_{0} /[\mathrm{NaBArF}]_{0} /[\mathrm{AIBN}]_{0} /[\mathrm{AN}]_{0}=1 / 1 / 20 / 500$. 
Table S12. $t \mathrm{BA}$ polymerization mediated by $\mathrm{Al}^{\mathrm{III}}($ tralen)Cl in $\mathrm{DMF}$ under the condition of $\left[\mathrm{Al}^{\mathrm{III}}(\text { tralen }) \mathrm{Cl}\right]_{0} /[\mathrm{NaBArF}]_{0} /[\mathrm{AIBN}]_{0} /[\mathrm{tBA}]_{0}=1 / 1 / 20 / 1000$ at $60^{\circ} \mathrm{C},[t \mathrm{BA}]_{0}=2.27 \mathrm{M}$.

\begin{tabular}{ccccc}
\hline $\begin{array}{c}\text { Time } \\
(\mathrm{min})\end{array}$ & $\begin{array}{c}\text { Conv. }^{\mathrm{a}} \\
(\%)\end{array}$ & $\begin{array}{c}M_{\mathrm{n}, \exp }{ }^{\mathrm{b}} \\
(\mathrm{g} / \mathrm{mol})\end{array}$ & $\begin{array}{c}M_{\mathrm{n}, \mathrm{th}^{\mathrm{c}}} \\
(\mathrm{g} / \mathrm{mol})\end{array}$ & PDI $^{\mathrm{b}}$ \\
\hline 10 & 86 & 52,200 & 98,000 & 1.96 \\
\hline
\end{tabular}

${ }^{\mathrm{a} C}$ Conversion was detected by ${ }^{1} \mathrm{H}$ NMR. ${ }^{\mathrm{b}} M_{\mathrm{n}}$ and PDI were determined by gel permeation chromatography (GPC) in THF with polystyrene as standard. ${ }^{\mathrm{c}} M_{\mathrm{n}, \mathrm{th}}=M_{\mathrm{w}(\text { mediator })}+M_{\mathrm{w}(t \mathrm{BA})} \times\left([t \mathrm{BA}]_{0} /\left[\mathrm{Al}^{\mathrm{III}}\right]_{0}\right) \times$ conv..

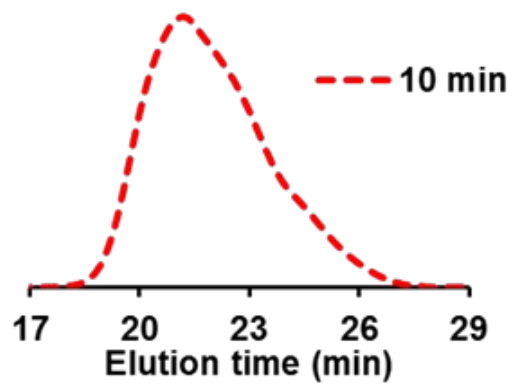

Figure S19. The GPC traces for $t \mathrm{BA}$ polymerization mediated by $\mathrm{Al}{ }^{\mathrm{III}}$ (tralen) $\mathrm{Cl}$ at $60^{\circ} \mathrm{C}$ in bulk under the following condition of $\left[\mathrm{Al}{ }^{\mathrm{III}}(\text { tralen}) \mathrm{Cl}\right]_{0} /[\mathrm{NaBArF}]_{0} /[\mathrm{AIBN}]_{0} /[t \mathrm{BA}]_{0}=1 / 1 / 20 / 1000$.

Table S13. Sty polymerization mediated by $\mathrm{Al}$ III $(\operatorname{tralen}) \mathrm{Cl}$ in $\mathrm{DMF}$ under the condition of $\left[\mathrm{Al}^{\mathrm{III}} \text { (tralen) } \mathrm{Cl}\right]_{0} /[\mathrm{NaBArF}]_{0} /[\mathrm{ABCN}]_{0} /[\mathrm{Sty}]_{0}=1 / 1 / 20 / 500$ at $60{ }^{\circ} \mathrm{C},[\mathrm{Sty}]_{0}=4.32 \mathrm{M}$.

\begin{tabular}{ccccc}
\hline Time $(\min )$ & $\begin{array}{c}\text { Conv. } \\
(\%)\end{array}$ & $\begin{array}{c}M_{\mathrm{n}, \exp }{ }^{\mathrm{b}} \\
(\mathrm{g} / \mathrm{mol})\end{array}$ & $\begin{array}{c}M_{\mathrm{n}, \mathrm{th}}{ }^{\mathrm{c}} \\
(\mathrm{g} / \mathrm{mol})\end{array}$ & PDI $^{\mathrm{b}}$ \\
\hline 180 & 20 & 35,200 & 21,700 & 1.75 \\
300 & 28 & 39,100 & 29,700 & 1.62 \\
360 & 36 & 37,300 & 37,700 & 1.69 \\
420 & 40 & 37,300 & 42,400 & 1.66 \\
480 & 45 & 39,400 & 47,700 & 1.66 \\
\hline
\end{tabular}

${ }^{a}$ Conversion was detected by ${ }^{1} \mathrm{H}$ NMR. ${ }^{\mathrm{b}} M_{\mathrm{n}}$ and PDI were determined by gel permeation chromatography (GPC) in THF with polystyrene as standard. ${ }^{\mathrm{c}} M_{\mathrm{n}, \mathrm{th}}=M_{\mathrm{w}(\text { mediator })}+M_{\mathrm{w}(\mathrm{Sty})} \times\left([\mathrm{Sty}]_{0} /\left[\mathrm{Al}^{\mathrm{III}}\right]_{0}\right) \times$ conv.. 

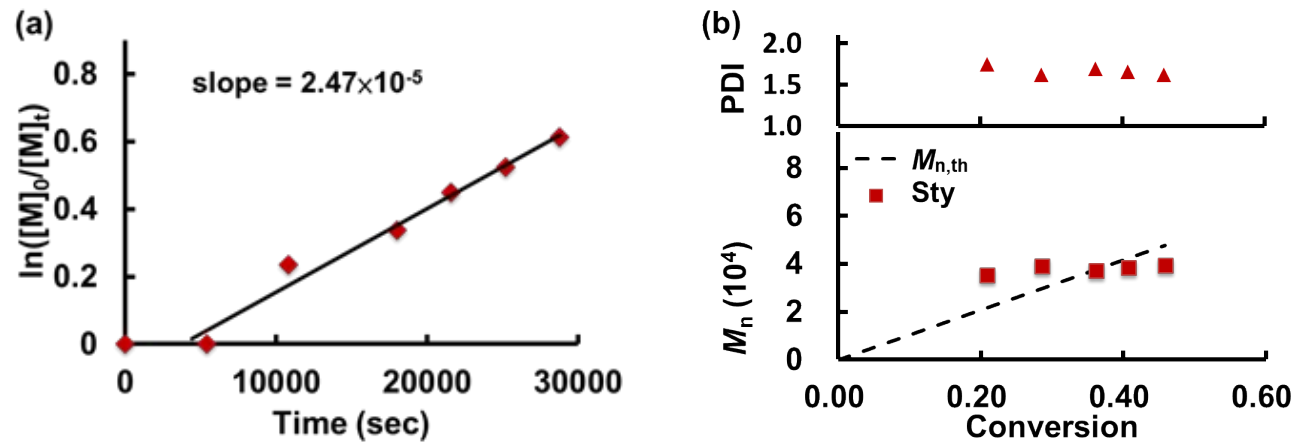

Figure S20. Sty polymerization mediated by $\mathrm{Al}{ }^{1 \mathrm{II}}($ tralen$) \mathrm{Cl}$ at $60{ }^{\circ} \mathrm{C}$ in $\mathrm{DMF}$ under the condition of $\left[\mathrm{Al}^{\mathrm{III}}(\right.$ tralen$\left.\left.) \mathrm{Cl}\right)\right]_{0} /[\mathrm{NaBArF}]_{0} /[\mathrm{ABCN}]_{0} /[\mathrm{Sty}]_{0}=1 / 1 / 20 / 500$ with $[\mathrm{Sty}]_{0}=4.32 \mathrm{M}$. (a) First-order kinetic plots. (b) The plots of $M_{\mathrm{n}}$ and PDI versus conversion.

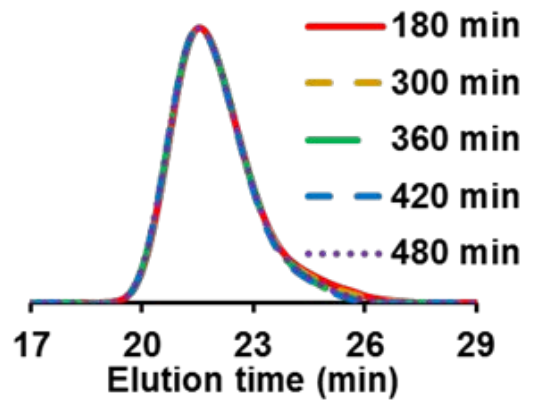

Figure S21. The GPC traces for Sty polymerization mediated by $\mathrm{Al}^{\mathrm{III}}\left(\right.$ tralen) $\mathrm{Cl}$ at $60{ }^{\circ} \mathrm{C}$ in bulk under the following condition of $\left[\mathrm{Al}{ }^{\mathrm{III}}(\text { tralen }) \mathrm{Cl}\right]_{0} /[\mathrm{NaBArF}]_{0} /[\mathrm{ABCN}]_{0} /[\mathrm{Sty}]_{0}=1 / 1 / 20 / 1000$.

Table S14. Free radical polymerization of vinyl monomers under the condition of $[\mathrm{AIBN}]_{0} /[\text { monomer }]_{0}=20 / 1000$ at $60{ }^{\circ} \mathrm{C}$ in DMF.

\begin{tabular}{cccccc}
\hline Entry & Monomer & Time $(\min )$ & Conv. $^{a}(\%)$ & $M_{\mathrm{n}}{ }^{b}(\mathrm{~g} / \mathrm{mol})$ & PDI $^{b}$ \\
\hline $1^{c}$ & VAc & 20 & 28 & 146200 & 2.90 \\
$2^{d}$ & NVP & 15 & 44 & 68200 & 4.09 \\
3 & MA & 5 & 87 & 107200 & 2.69 \\
4 & tBA & 6 & 35 & 166700 & 1.44 \\
$5^{d}$ & AN & 10 & 48 & 174900 & 6.24 \\
$6^{e}$ & Sty & 20 & 50 & 19600 & 2.05 \\
\hline
\end{tabular}

${ }^{a}$ Conversion was detected by ${ }^{1} \mathrm{H}$ NMR. ${ }^{b} M_{\mathrm{n}}$ and PDI were determined by gel permeation chromatography (GPC) in THF with polystyrene as standard. ${ }^{c}$ In bulk. ${ }^{d} M_{\mathrm{n}}$ and PDI were determined by gel permeation chromatography (GPC) in DMF with poly(ethylene glycol) (PEG) as standard corrected by the Mark-Houwink equation. ${ }^{e} 1-1$ azobis(cyclohexanecarbonitrile (ABCN) as radical initiator. 


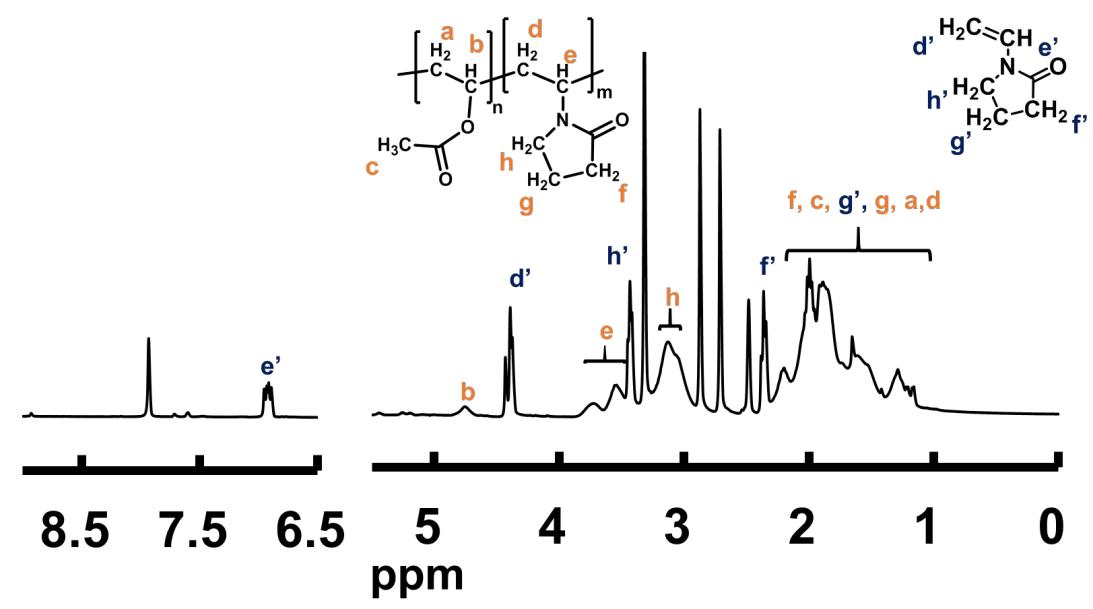

Figure S22. ${ }^{1} \mathrm{H}$ NMR spectrum of PVAc- $b$-PNVP block copolymer synthesized from a PVAc-Al ${ }^{\mathrm{III}}$ (tralen)BArF macro-initiator in DMSO- $d_{6}$.

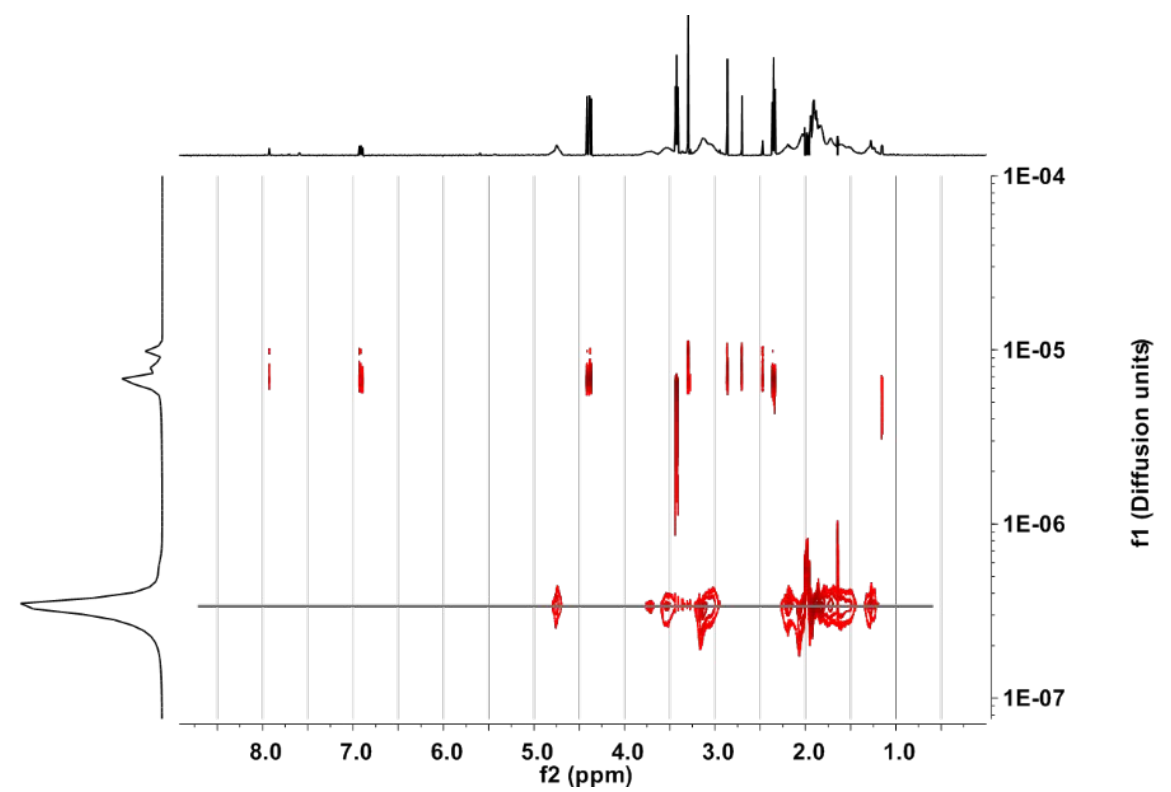

Figure S23. DOSY-NMR spectrum of PVAc- $b$-PNVP block copolymer synthesized from a PVAc-AlIII(tralen)BArF macroinitiator in DMSO- $d_{6}$. 

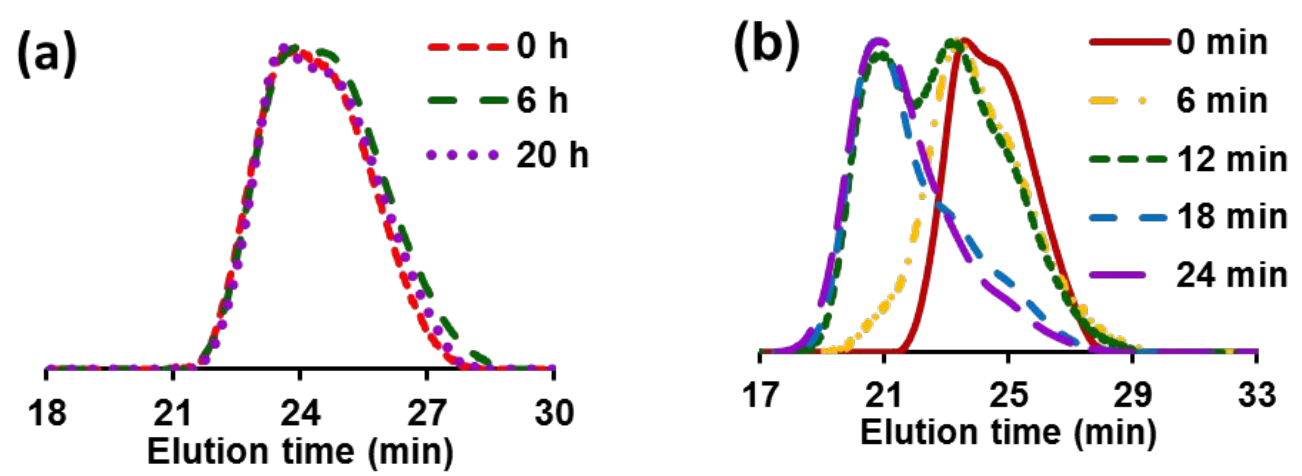

Figure S24. The GPC traces of VAc polymerization mediated by PVAc-Al ${ }^{\mathrm{II}}($ tralen)BArF under the condition of (a) [PVAc$\mathrm{Al}^{\mathrm{III}}$ (tralen)BArF $]_{0} /[\mathrm{AIBN}]_{0} /[\mathrm{VAc}]_{0}=1 / 0 / 1000$ and (b) $\left[\mathrm{PVAc}-\mathrm{Al}^{\mathrm{III}} \text { (tralen)BArF }\right]_{0} /[\mathrm{AIBN}]_{0} /[\mathrm{VAc}]_{0}=1 / 20 / 500$ in bulk at 60 ${ }^{\circ} \mathrm{C}$. The organo-aluminum complex PVAc-Al ${ }^{\mathrm{III}}$ (tralen)BArF was synthesis under the condition of $\left[\mathrm{Al}^{\mathrm{III}} \text { (tralen) } \mathrm{Cl}\right]_{0} /[\mathrm{NaBArF}]_{0} /[\mathrm{AIBN}]_{0} /[\mathrm{VAc}]_{0}=1 / 1 / 20 / 200$ at $60{ }^{\circ} \mathrm{C}$ in bulk for 300 minutes with conversion equal to $19.3 \%$ with $M_{\mathrm{n}}$ equal to $4,800 \mathrm{~g} / \mathrm{mol}$ and PDI equal to 1.53 .

\section{Analysis for the structure of the $\omega$ chain ends}

GPC traces for PVAc were measured by both refractive index and UV-visible detectors (Figure S25). Polymer chain end with the organo-aluminum chromophore showed a strong UV-visible absorption band around $354 \mathrm{~nm}$. The GPC traces of PVAc measured by RID and UV-vis detectors were nearly the same, except for a delay in RID signal due to the series connection of the equipment. The elution curves indicated that each polymer chain contained aluminum complex at the $\omega$ end.

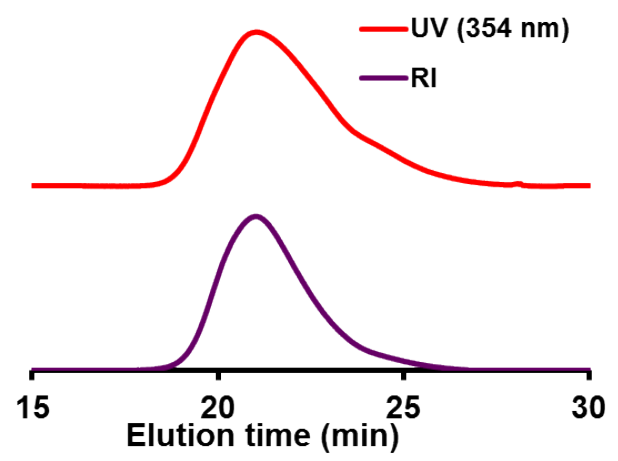

Figure S25. GPC traces for VAc polymerization mediated by $\mathrm{Al}{ }^{\mathrm{III}}($ tralen$) \mathrm{Cl}$ under the condition of $\left[\mathrm{Al}{ }^{\mathrm{III}} \text { (tralen) } \mathrm{Cl}\right]_{0} /[\mathrm{NaBArF}]_{0} /[\mathrm{AIBN}]_{0} /[\mathrm{VAc}]_{0}=1 / 1 / 20 / 1000$ at $60^{\circ} \mathrm{C}$ in bulk $\left(M_{\mathrm{n}, \mathrm{GPC}}=45,300 \mathrm{~g} / \mathrm{mol}, \mathrm{PDI}=2.17\right)$. The red line corresponded to the UV-visible (354 nm) detection trace, and the purple line corresponded the refractive index detection trace. 


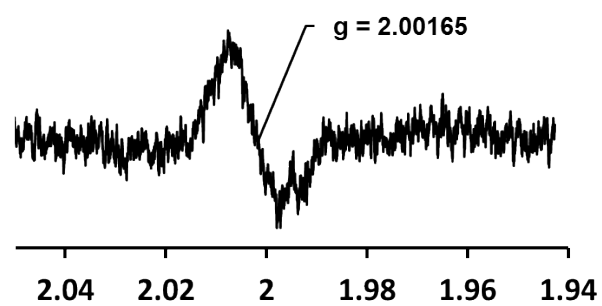

Figure S26. EPR spectrum of PVAc-Al ${ }^{\mathrm{III}}($ tralen$) \mathrm{Cl}$ in DMSO- $d_{6}$ at room temperature. $\mathrm{PVAc}-\mathrm{Al}{ }^{\mathrm{II}}($ tralen$) \mathrm{Cl}$ produced by $\mathrm{Al}^{\mathrm{III}}$ (tralen)Cl mediated VAc polymerization under the condition of $\left[\mathrm{Al}{ }^{\mathrm{III}}(\text { tralen }) \mathrm{Cl}\right]_{0} /[\mathrm{AIBN}]_{0} /[\mathrm{VAc}]_{0}=1 / 20 / 1000$ at $60{ }^{\circ} \mathrm{C}$ in bulk. The $M_{\mathrm{n}}$ approached $42600 \mathrm{~g} / \mathrm{mol}$ with PDI eqaul to 2.06 when the conversion was $45 \%$ in 360 minutes.

\section{Crystallographic data of $\mathrm{Al}^{\mathrm{III}}($ tralen$) \mathrm{Cl}$}

Table S15. Crystal data and structure refinement for $\mathrm{Al}^{\mathrm{III}}(\operatorname{tralen}) \mathrm{Cl}$

\begin{tabular}{|c|c|}
\hline Identification code & 119Al_0m \\
\hline Empirical formula & $\mathrm{C}_{20} \mathrm{H}_{14} \mathrm{AlClN}_{2} \mathrm{O}_{2}$ \\
\hline Formula weight & 376.76 \\
\hline Temperature/K & 150.0 \\
\hline Crystal system & monoclinic \\
\hline Space group & $\mathrm{P} 2_{1} / \mathrm{n}$ \\
\hline $\mathrm{a} / \AA$ & $14.3671(9)$ \\
\hline $\mathrm{b} / \AA$ & $13.5142(9)$ \\
\hline $\mathrm{c} / \AA$ & $18.3458(10)$ \\
\hline$\alpha /{ }^{\circ}$ & 90 \\
\hline$\beta /{ }^{\circ}$ & $112.194(2)$ \\
\hline$\gamma /{ }^{\circ}$ & 90 \\
\hline Volume $/ \AA^{3}$ & $3298.1(4)$ \\
\hline Z & 8 \\
\hline$\rho_{\text {calc }} \mathrm{g} / \mathrm{cm}^{3}$ & 1.518 \\
\hline$\mu / \mathrm{mm}^{-1}$ & 0.303 \\
\hline $\mathrm{F}(000)$ & 1552.0 \\
\hline Crystal size $/ \mathrm{mm}^{3}$ & $0.12 \times 0.08 \times 0.05$ \\
\hline Radiation & $\operatorname{MoK} \alpha(\lambda=0.71073)$ \\
\hline $2 \Theta$ range for data collection $/{ }^{\circ}$ & 4.546 to 51.996 \\
\hline Index ranges & $-17 \leq \mathrm{h} \leq 17,-16 \leq \mathrm{k} \leq 16,-20 \leq 1 \leq 22$ \\
\hline Reflections collected & 33395 \\
\hline Independent reflections & $6472\left[\mathrm{R}_{\mathrm{int}}=0.0981, \mathrm{R}_{\text {sigma }}=0.0839\right]$ \\
\hline Data/restraints/parameters & $6472 / 0 / 469$ \\
\hline Goodness-of-fit on $\mathrm{F}^{2}$ & 1.043 \\
\hline Final $R$ indexes $[I>=2 \sigma(I)]$ & $\mathrm{R}_{1}=0.0684, \mathrm{wR}_{2}=0.1044$ \\
\hline Final $\mathrm{R}$ indexes [all data] & $\mathrm{R}_{1}=0.1315, \mathrm{wR}_{2}=0.1187$ \\
\hline Largest diff. peak/hole / e $\AA^{-3}$ & $0.36 /-0.36$ \\
\hline
\end{tabular}


Table S16. Fractional Atomic Coordinates $\left(\times 10^{4}\right)$ and Equivalent Isotropic Displacement Parameters $\left(\AA^{2} \times 10^{3}\right)$ for $\mathrm{Al}\left(\right.$ tralen) $\mathrm{Cl} . \mathrm{U}_{\text {eq }}$ is defined as $1 / 3$ of of the trace of the orthogonalised $\mathrm{U}_{\mathrm{IJ}}$ tensor.

\begin{tabular}{|c|c|c|c|c|}
\hline Atom & $x$ & $y$ & $z$ & $\mathrm{U}(\mathrm{eq})$ \\
\hline Cl1 & $8738.5(7)$ & $7237.9(7)$ & $4208.8(6)$ & $29.5(2)$ \\
\hline Al1 & $7113.6(8)$ & $7428.3(8)$ & $3661.0(6)$ & $22.0(3)$ \\
\hline $\mathrm{O} 1$ & 6732.2(19) & $6732.9(19)$ & $2744.8(14)$ & $27.8(6)$ \\
\hline $\mathrm{O} 2$ & $6916.5(19)$ & $8644.6(18)$ & $3215.3(14)$ & $25.7(6)$ \\
\hline N1 & $6536(2)$ & $6320(2)$ & $3991.3(17)$ & $20.6(7)$ \\
\hline N2 & $6702(2)$ & $8082(2)$ & $4427.4(17)$ & $21.0(7)$ \\
\hline $\mathrm{C} 1$ & $6502(3)$ & $10285(3)$ & $3251(2)$ & $26.8(9)$ \\
\hline $\mathrm{C} 2$ & $6247(3)$ & 11174(3) & $3511(2)$ & $32.2(10)$ \\
\hline $\mathrm{C} 3$ & $6028(3)$ & $11370(3)$ & $4164(2)$ & $33.7(10)$ \\
\hline $\mathrm{C} 4$ & $5976(3)$ & 10673(3) & $4710(2)$ & $31.6(10)$ \\
\hline $\mathrm{C} 5$ & $6162(3)$ & $9677(3)$ & $4760(2)$ & $26.8(9)$ \\
\hline C6 & $6508(3)$ & $9043(3)$ & $4306(2)$ & $22.5(9)$ \\
\hline $\mathrm{C} 7$ & $6650(3)$ & 9354(3) & $3581(2)$ & $23.8(9)$ \\
\hline $\mathrm{C} 8$ & $6599(3)$ & $7430(3)$ & 4996(2) & $22.0(9)$ \\
\hline C9 & $6660(3)$ & $7653(3)$ & $5755(2)$ & 24.9(9) \\
\hline $\mathrm{C} 10$ & $6623(3)$ & $6898(3)$ & $6254(2)$ & $28.4(10)$ \\
\hline C11 & $6538(3)$ & $5927(3)$ & $6014(2)$ & 26.3(9) \\
\hline $\mathrm{C} 12$ & $6483(3)$ & $5692(3)$ & $5261(2)$ & $23.8(9)$ \\
\hline $\mathrm{C} 13$ & $6510(3)$ & $6432(3)$ & 4749(2) & 21.2(9) \\
\hline $\mathrm{C} 14$ & $6210(3)$ & $5586(3)$ & $3475(2)$ & $20.9(8)$ \\
\hline $\mathrm{C} 15$ & $5684(3)$ & $4736(3)$ & $3556(2)$ & $24.6(9)$ \\
\hline $\mathrm{C} 16$ & $5442(3)$ & $3895(3)$ & $3116(2)$ & 28.1(9) \\
\hline $\mathrm{C} 17$ & $5615(3)$ & $3625(3)$ & $2442(2)$ & 28.3(9) \\
\hline $\mathrm{C} 18$ & $5972(3)$ & $4226(3)$ & $2009(2)$ & $28.1(10)$ \\
\hline $\mathrm{C} 19$ & $6260(3)$ & $5218(3)$ & $2130(2)$ & $25.0(9)$ \\
\hline $\mathrm{C} 20$ & 6393(3) & $5830(3)$ & $2760(2)$ & $22.7(9)$ \\
\hline $\mathrm{Cl} 2$ & $6274.1(7)$ & $3159.1(7)$ & $5813.3(6)$ & $30.3(2)$ \\
\hline $\mathrm{A} 12$ & 7895.1(9) & $2956.9(8)$ & $6356.4(6)$ & $22.5(3)$ \\
\hline $\mathrm{O} 3$ & $8089.6(19)$ & 1727.1(18) & $6790.8(14)$ & $25.4(6)$ \\
\hline $\mathrm{O} 4$ & $8290.3(19)$ & $3637.7(19)$ & $7278.3(14)$ & $27.5(6)$ \\
\hline N3 & $8492(2)$ & 4061(2) & $6032.7(17)$ & $21.6(7)$ \\
\hline N4 & $8282(2)$ & $2306(2)$ & $5573.2(16)$ & $22.5(7)$ \\
\hline $\mathrm{C} 21$ & $8645(3)$ & $4530(3)$ & $7270(2)$ & $22.8(9)$ \\
\hline $\mathrm{C} 22$ & $8795(3)$ & $5143(3)$ & $7910(2)$ & $24.5(9)$ \\
\hline $\mathrm{C} 23$ & 9099(3) & $6127(3)$ & $8043(2)$ & 26.6(9) \\
\hline $\mathrm{C} 24$ & $9447(3)$ & $6734(3)$ & $7604(2)$ & $28.7(9)$ \\
\hline $\mathrm{C} 25$ & $9605(3)$ & $6478(3)$ & $6922(2)$ & 28.6(9) \\
\hline $\mathrm{C} 26$ & 9353(3) & $5636(3)$ & $6476(2)$ & $23.8(9)$ \\
\hline $\mathrm{C} 27$ & $8823(3)$ & 4790(3) & $6556(2)$ & $20.7(8)$ \\
\hline $\mathrm{C} 28$ & $8502(3)$ & $3956(3)$ & $5270(2)$ & $21.5(8)$ \\
\hline
\end{tabular}




$\begin{array}{lllll}\mathrm{C} 29 & 8534(3) & 4701(3) & 4762(2) & 24.4(9) \\ \mathrm{C} 30 & 8461(3) & 4471(3) & 4004(2) & 28.1(9) \\ \mathrm{C} 31 & 8329(3) & 3505(3) & 3747(2) & 27.1(9) \\ \mathrm{C} 32 & 8281(3) & 2750(3) & 4243(2) & 25.5(9) \\ \mathrm{C} 33 & 8376(3) & 2958(3) & 5007(2) & 21.6(9) \\ \mathrm{C} 34 & 1334(3) & 5683(2) & 21.9(9) \\ \mathrm{C} 35 & 8454(3) & 715(3) & 5214(2) & 26.5(9) \\ \mathrm{C} 36 & 8793(3) & -289(3) & 5248(2) & 30.1(10) \\ \mathrm{C} 37 & 8951(3) & -996(3) & 5775(2) & 29.8(10) \\ \text { C38 } & 8865(3) & -817(3) & 6426(2) & 27.7(9) \\ \text { C39 } & 8641(3) & 64(3) & 6702(2) & 25.8(9) \\ \text { C40 } & 8412(3) & 1009(3) & 6396(2) & 21.9(8)\end{array}$

Table S17. Anisotropic Displacement Parameters $\left(\AA^{\left.2 \times 10^{3}\right)}\right.$ for $\mathrm{Al}{ }^{\mathrm{III}}($ tralen$) \mathrm{Cl}$. The Anisotropic displacement factor exponent takes the form: $-2 \pi^{2}\left[h^{2} a * 2 U_{11}+2 h k a * b * U_{12}+\ldots\right]$.

\begin{tabular}{|c|c|c|c|c|c|c|}
\hline Atom & $\mathrm{U}_{11}$ & $\mathbf{U}_{22}$ & $\mathbf{U}_{33}$ & $\mathbf{U}_{23}$ & $\mathbf{U}_{13}$ & $\mathrm{U}_{12}$ \\
\hline $\mathrm{Cl1}$ & $29.2(6)$ & $28.1(6)$ & $33.5(6)$ & $4.9(4)$ & $14.5(5)$ & $1.2(4)$ \\
\hline Al1 & $29.0(7)$ & $21.5(7)$ & $18.8(6)$ & $2.0(5)$ & $12.8(5)$ & $0.5(5)$ \\
\hline $\mathrm{O} 1$ & $38.2(17)$ & $29.7(17)$ & $18.7(14)$ & $1.9(11)$ & $14.6(13)$ & $-1.6(12)$ \\
\hline $\mathrm{O} 2$ & $34.4(16)$ & $26.4(16)$ & $18.6(14)$ & $1.6(11)$ & $12.6(12)$ & $1.5(12)$ \\
\hline N1 & $28.1(18)$ & $20.2(18)$ & $16.2(16)$ & $1.7(13)$ & $11.4(14)$ & $0.9(13)$ \\
\hline $\mathrm{N} 2$ & $27.8(19)$ & $20(2)$ & $16.2(16)$ & $-0.9(13)$ & $9.5(14)$ & $-1.3(13)$ \\
\hline $\mathrm{C} 1$ & $26(2)$ & $28(3)$ & $26(2)$ & $2.1(18)$ & $8.5(18)$ & $-2.0(17)$ \\
\hline $\mathrm{C} 2$ & $32(2)$ & $27(3)$ & $32(2)$ & $2.2(18)$ & $5(2)$ & $-2.0(18)$ \\
\hline $\mathrm{C} 3$ & $38(3)$ & $22(2)$ & $37(3)$ & $-3.2(19)$ & $8(2)$ & $3.5(18)$ \\
\hline $\mathrm{C} 4$ & $34(2)$ & $30(3)$ & $28(2)$ & $-9.2(19)$ & $9(2)$ & $0.9(18)$ \\
\hline $\mathrm{C} 5$ & $31(2)$ & $30(3)$ & $21(2)$ & $-3.4(17)$ & $11.6(18)$ & $-1.1(18)$ \\
\hline C6 & $18(2)$ & $23(2)$ & $24(2)$ & $-2.4(17)$ & $4.4(17)$ & $-5.6(16)$ \\
\hline $\mathrm{C} 7$ & $24(2)$ & $26(3)$ & $19(2)$ & $1.8(17)$ & $6.0(17)$ & $-2.6(17)$ \\
\hline $\mathrm{C} 8$ & $20(2)$ & $27(2)$ & $20(2)$ & $1.0(16)$ & $9.0(17)$ & $1.4(16)$ \\
\hline C9 & $29(2)$ & $26(2)$ & $23(2)$ & $-4.4(17)$ & $12.9(18)$ & $0.3(17)$ \\
\hline $\mathrm{C} 10$ & $32(2)$ & $39(3)$ & $16(2)$ & $-0.6(18)$ & $12.7(18)$ & $-3.0(18)$ \\
\hline $\mathrm{C} 11$ & $29(2)$ & $33(3)$ & $19(2)$ & $8.2(17)$ & $12.8(18)$ & $2.4(18)$ \\
\hline $\mathrm{C} 12$ & $22(2)$ & $26(2)$ & $22(2)$ & $1.8(17)$ & 7.1(17) & $-1.6(16)$ \\
\hline $\mathrm{C} 13$ & $21(2)$ & $27(2)$ & $17(2)$ & $2.9(16)$ & $8.7(17)$ & $3.1(16)$ \\
\hline $\mathrm{C} 14$ & $21(2)$ & $24(2)$ & $19(2)$ & $0.5(16)$ & $8.6(17)$ & $4.0(16)$ \\
\hline $\mathrm{C} 15$ & $28(2)$ & $28(2)$ & $19(2)$ & $0.4(17)$ & $10.5(17)$ & $-1.4(17)$ \\
\hline $\mathrm{C} 16$ & $27(2)$ & $31(3)$ & $24(2)$ & $1.4(18)$ & $6.6(18)$ & $-4.6(18)$ \\
\hline $\mathrm{C} 17$ & $27(2)$ & $24(2)$ & $29(2)$ & $-1.2(17)$ & $3.9(19)$ & $1.6(17)$ \\
\hline $\mathrm{C} 18$ & $24(2)$ & $31(3)$ & $27(2)$ & $-6.8(18)$ & $7.7(18)$ & $8.1(18)$ \\
\hline $\mathrm{C} 19$ & $27(2)$ & $33(3)$ & $18(2)$ & $-0.5(17)$ & $11.5(17)$ & $4.7(18)$ \\
\hline $\mathrm{C} 20$ & $23(2)$ & $24(2)$ & $22(2)$ & $5.2(17)$ & $9.0(17)$ & $2.6(16)$ \\
\hline
\end{tabular}




\begin{tabular}{|c|c|c|c|c|c|c|}
\hline $\mathrm{Cl} 2$ & $29.1(6)$ & $28.0(6)$ & $35.1(6)$ & $5.7(4)$ & $13.5(5)$ & $1.3(4)$ \\
\hline $\mathrm{A} 12$ & $28.4(7)$ & 24.7(7) & $17.0(6)$ & $0.5(5)$ & $11.6(5)$ & $-1.5(5)$ \\
\hline $\mathrm{O} 3$ & $33.9(16)$ & $24.1(16)$ & $21.9(14)$ & $2.6(11)$ & $14.8(12)$ & $1.6(11)$ \\
\hline $\mathrm{O} 4$ & $39.2(17)$ & $26.3(17)$ & $21.2(14)$ & $-0.1(11)$ & $16.3(13)$ & $-9.1(12)$ \\
\hline N3 & $24.7(18)$ & $27.4(19)$ & $16.2(16)$ & $0.2(14)$ & $11.8(14)$ & $0.2(14)$ \\
\hline N4 & $25.0(18)$ & $26(2)$ & $16.5(16)$ & $-0.3(13)$ & $8.3(14)$ & $-1.6(14)$ \\
\hline $\mathrm{C} 21$ & $17(2)$ & $31(3)$ & $19(2)$ & $1.9(17)$ & $5.7(16)$ & $4.7(17)$ \\
\hline $\mathrm{C} 22$ & $25(2)$ & $31(3)$ & $20(2)$ & $0.2(17)$ & $11.2(17)$ & $-0.2(17)$ \\
\hline $\mathrm{C} 23$ & $27(2)$ & $30(3)$ & $22(2)$ & $-2.3(17)$ & $8.2(18)$ & $7.8(18)$ \\
\hline $\mathrm{C} 24$ & $28(2)$ & $25(2)$ & $27(2)$ & $-4.1(18)$ & $3.9(18)$ & $2.8(17)$ \\
\hline $\mathrm{C} 25$ & $31(2)$ & $22(2)$ & $30(2)$ & $2.9(18)$ & $8.9(19)$ & $-0.4(17)$ \\
\hline $\mathrm{C} 26$ & $23(2)$ & $28(2)$ & $21(2)$ & $2.5(17)$ & $8.2(17)$ & $0.9(17)$ \\
\hline $\mathrm{C} 27$ & $20(2)$ & $25(2)$ & $16.2(19)$ & $0.4(16)$ & $6.5(16)$ & $0.9(16)$ \\
\hline $\mathrm{C} 28$ & $20(2)$ & $29(2)$ & $15.8(19)$ & $3.4(17)$ & $7.5(16)$ & $-0.4(16)$ \\
\hline $\mathrm{C} 29$ & $23(2)$ & $27(2)$ & $23(2)$ & $2.7(17)$ & $8.9(17)$ & $1.8(16)$ \\
\hline C30 & $30(2)$ & $34(3)$ & $22(2)$ & $6.7(18)$ & $11.3(18)$ & $-0.2(18)$ \\
\hline $\mathrm{C} 31$ & $25(2)$ & $43(3)$ & $15(2)$ & $0.4(18)$ & $9.3(18)$ & $1.4(18)$ \\
\hline $\mathrm{C} 32$ & $26(2)$ & $30(2)$ & $21(2)$ & $-2.1(17)$ & $8.3(17)$ & $-0.5(17)$ \\
\hline C33 & $24(2)$ & $25(2)$ & $17(2)$ & $1.2(16)$ & $8.9(17)$ & $-2.7(16)$ \\
\hline $\mathrm{C} 34$ & $21(2)$ & $25(2)$ & $19(2)$ & $0.1(16)$ & $6.3(17)$ & $-2.8(16)$ \\
\hline $\mathrm{C} 35$ & $26(2)$ & $35(3)$ & $20(2)$ & $-1.3(17)$ & $10.2(17)$ & $-2.7(18)$ \\
\hline $\mathrm{C} 36$ & $26(2)$ & $34(3)$ & $25(2)$ & $-6.0(19)$ & $2.9(18)$ & $1.7(18)$ \\
\hline C37 & $29(2)$ & $24(2)$ & $30(2)$ & $-6.4(18)$ & $3.3(19)$ & $1.3(17)$ \\
\hline C38 & $28(2)$ & $19(2)$ & $28(2)$ & $1.3(17)$ & $0.9(18)$ & $0.2(17)$ \\
\hline C39 & $26(2)$ & $27(3)$ & $22(2)$ & $5.1(17)$ & $7.7(18)$ & $-1.0(17)$ \\
\hline $\mathrm{C} 40$ & $20(2)$ & $23(2)$ & $20(2)$ & $-1.8(17)$ & $5.3(17)$ & $-1.8(16)$ \\
\hline
\end{tabular}

Table S18. Bond Lengths for $\mathrm{Al}^{\mathrm{III}}($ tralen$) \mathrm{Cl}$.

\begin{tabular}{|c|c|c|c|c|c|}
\hline Atom & Atom & Length/Å & Atom & Atom & Length $/ \AA ̊ \AA$ \\
\hline $\mathrm{Cl1}$ & Al1 & $2.1803(15)$ & $\mathrm{Cl} 2$ & A12 & $2.1766(15)$ \\
\hline Al1 & $\mathrm{O} 1$ & $1.820(3)$ & $\mathrm{A} 12$ & $\mathrm{O} 3$ & $1.819(3)$ \\
\hline Al1 & $\mathrm{O} 2$ & $1.810(3)$ & $\mathrm{A} 12$ & $\mathrm{O} 4$ & $1.818(3)$ \\
\hline Al1 & N1 & $1.918(3)$ & $\mathrm{A} 12$ & N3 & $1.923(3)$ \\
\hline Al1 & $\mathrm{N} 2$ & $1.933(3)$ & $\mathrm{A} 12$ & N4 & $1.937(3)$ \\
\hline $\mathrm{O} 1$ & $\mathrm{C} 20$ & $1.318(4)$ & $\mathrm{O} 3$ & $\mathrm{C} 40$ & $1.320(4)$ \\
\hline $\mathrm{O} 2$ & $\mathrm{C} 7$ & $1.307(4)$ & $\mathrm{O} 4$ & $\mathrm{C} 21$ & $1.311(4)$ \\
\hline N1 & $\mathrm{C} 13$ & $1.412(4)$ & N3 & $\mathrm{C} 27$ & $1.331(4)$ \\
\hline N1 & $\mathrm{C} 14$ & $1.329(4)$ & N3 & $\mathrm{C} 28$ & $1.412(4)$ \\
\hline $\mathrm{N} 2$ & C6 & $1.329(4)$ & N4 & $\mathrm{C} 33$ & $1.407(4)$ \\
\hline $\mathrm{N} 2$ & $\mathrm{C} 8$ & $1.415(4)$ & N4 & $\mathrm{C} 34$ & $1.338(4)$ \\
\hline $\mathrm{C} 1$ & $\mathrm{C} 2$ & $1.393(5)$ & $\mathrm{C} 21$ & $\mathrm{C} 22$ & $1.385(5)$ \\
\hline $\mathrm{C} 1$ & $\mathrm{C} 7$ & $1.377(5)$ & $\mathrm{C} 21$ & $\mathrm{C} 27$ & $1.470(5)$ \\
\hline
\end{tabular}




$\begin{array}{llllll}\mathrm{C} 2 & \mathrm{C} 3 & 1.373(5) & \mathrm{C} 22 & \mathrm{C} 23 & 1.393(5) \\ \mathrm{C} 3 & \mathrm{C} 4 & 1.398(6) & \mathrm{C} 23 & \mathrm{C} 24 & 1.369(5) \\ \mathrm{C} 4 & \mathrm{C} 5 & 1.368(5) & \mathrm{C} 24 & \mathrm{C} 25 & 1.397(5) \\ \mathrm{C} 5 & \mathrm{C} 6 & 1.410(5) & \mathrm{C} 25 & \mathrm{C} 26 & 1.367(5) \\ \mathrm{C} 6 & \mathrm{C} 7 & 1.481(5) & \mathrm{C} 26 & \mathrm{C} 27 & 1.412(5) \\ \mathrm{C} 8 & \mathrm{C} 9 & 1.395(5) & \mathrm{C} 28 & \mathrm{C} 29 & 1.384(5) \\ \mathrm{C} 8 & \mathrm{C} 13 & 1.413(5) & \mathrm{C} 28 & \mathrm{C} 33 & 1.421(5) \\ \mathrm{C} 9 & \mathrm{C} 10 & 1.385(5) & \mathrm{C} 29 & \mathrm{C} 30 & 1.390(5) \\ \mathrm{C} 10 & \mathrm{C} 11 & 1.375(5) & \mathrm{C} 30 & \mathrm{C} 31 & 1.377(5) \\ \mathrm{C} 11 & \mathrm{C} 12 & 1.389(5) & \mathrm{C} 31 & \mathrm{C} 32 & 1.387(5) \\ \mathrm{C} 12 & \mathrm{C} 13 & 1.383(5) & \mathrm{C} 32 & \mathrm{C} 33 & 1.383(5) \\ \mathrm{C} 14 & \mathrm{C} 15 & 1.413(5) & \mathrm{C} 34 & \mathrm{C} 35 & 1.412(5) \\ \mathrm{C} 14 & \mathrm{C} 20 & 1.470(5) & \mathrm{C} 34 & \mathrm{C} 40 & 1.467(5) \\ \mathrm{C} 15 & \mathrm{C} 16 & 1.361(5) & \mathrm{C} 35 & \mathrm{C} 36 & 1.373(5) \\ \mathrm{C} 16 & \mathrm{C} 17 & 1.398(5) & \mathrm{C} 36 & \mathrm{C} 37 & 1.397(5) \\ \mathrm{C} 17 & \mathrm{C} 18 & 1.365(5) & \mathrm{C} 37 & \mathrm{C} 38 & 1.370(5) \\ \mathrm{C} 18 & \mathrm{C} 19 & 1.395(5) & \mathrm{C} 38 & \mathrm{C} 39 & 1.383(5) \\ \mathrm{C} 19 & \mathrm{C} 20 & 1.376(5) & \mathrm{C} 39 & \mathrm{C} 40 & 1.380(5)\end{array}$

Table S19. Bond Angles for $\mathrm{Al}^{\mathrm{III}}$ (tralen)Cl.

\begin{tabular}{|c|c|c|c|c|c|c|c|}
\hline Atom & Atom & Atom & Angle $/^{\circ}$ & Atom & Atom & Atom & Angle ${ }^{\circ}$ \\
\hline $\mathrm{O} 1$ & Al1 & $\mathrm{Cl1}$ & $105.14(10)$ & $\mathrm{O} 3$ & $\mathrm{~A} 12$ & $\mathrm{Cl} 2$ & $106.02(10)$ \\
\hline $\mathrm{O} 1$ & Al1 & N1 & $82.73(12)$ & $\mathrm{O} 3$ & $\mathrm{~A} 12$ & N3 & $146.26(13)$ \\
\hline $\mathrm{O} 1$ & Al1 & $\mathrm{N} 2$ & $147.10(13)$ & $\mathrm{O} 3$ & $\mathrm{~A} 12$ & N4 & $82.55(12)$ \\
\hline $\mathrm{O} 2$ & Al1 & $\mathrm{Cl1}$ & $105.84(10)$ & $\mathrm{O} 4$ & $\mathrm{~A} 12$ & $\mathrm{Cl} 2$ & $105.46(10)$ \\
\hline $\mathrm{O} 2$ & Al1 & $\mathrm{O} 1$ & $96.35(12)$ & $\mathrm{O} 4$ & $\mathrm{~A} 12$ & $\mathrm{O} 3$ & $96.46(12)$ \\
\hline $\mathrm{O} 2$ & Al1 & N1 & $147.05(13)$ & $\mathrm{O} 4$ & $\mathrm{~A} 12$ & N3 & $82.86(12)$ \\
\hline $\mathrm{O} 2$ & Al1 & $\mathrm{N} 2$ & $82.80(12)$ & $\mathrm{O} 4$ & $\mathrm{~A} 12$ & N4 & $147.55(13)$ \\
\hline N1 & Al1 & $\mathrm{Cl1}$ & $106.16(10)$ & N3 & A12 & $\mathrm{Cl} 2$ & $106.63(10)$ \\
\hline N1 & Al1 & $\mathrm{N} 2$ & $80.74(13)$ & N3 & $\mathrm{A} 12$ & N4 & $80.60(13)$ \\
\hline N2 & Al1 & $\mathrm{Cl1}$ & $106.71(10)$ & N4 & $\mathrm{A} 12$ & $\mathrm{Cl} 2$ & $105.95(10)$ \\
\hline $\mathrm{C} 20$ & $\mathrm{O} 1$ & Al1 & $116.3(2)$ & $\mathrm{C} 40$ & $\mathrm{O} 3$ & Al2 & $117.4(2)$ \\
\hline $\mathrm{C} 7$ & $\mathrm{O} 2$ & Al1 & $117.4(2)$ & $\mathrm{C} 21$ & $\mathrm{O} 4$ & $\mathrm{~A} 12$ & $116.2(2)$ \\
\hline $\mathrm{C} 13$ & N1 & Al1 & $113.7(2)$ & $\mathrm{C} 27$ & N3 & Al2 & $115.5(2)$ \\
\hline $\mathrm{C} 14$ & N1 & Al1 & $115.8(2)$ & $\mathrm{C} 27$ & $\mathrm{~N} 3$ & $\mathrm{C} 28$ & $130.9(3)$ \\
\hline $\mathrm{C} 14$ & N1 & $\mathrm{C} 13$ & $130.4(3)$ & $\mathrm{C} 28$ & N3 & $\mathrm{A} 12$ & $113.6(2)$ \\
\hline C6 & $\mathrm{N} 2$ & Al1 & $115.3(2)$ & $\mathrm{C} 33$ & N4 & $\mathrm{A} 12$ & $113.5(2)$ \\
\hline C6 & N2 & $\mathrm{C} 8$ & $131.2(3)$ & $\mathrm{C} 34$ & N4 & $\mathrm{A} 12$ & $115.0(2)$ \\
\hline $\mathrm{C} 8$ & $\mathrm{~N} 2$ & Al1 & $113.4(2)$ & $\mathrm{C} 34$ & N4 & $\mathrm{C} 33$ & $131.4(3)$ \\
\hline $\mathrm{C} 7$ & $\mathrm{C} 1$ & $\mathrm{C} 2$ & $130.9(4)$ & $\mathrm{O} 4$ & $\mathrm{C} 21$ & $\mathrm{C} 22$ & $118.5(3)$ \\
\hline $\mathrm{C} 3$ & $\mathrm{C} 2$ & $\mathrm{C} 1$ & $129.9(4)$ & $\mathrm{O} 4$ & $\mathrm{C} 21$ & $\mathrm{C} 27$ & $115.4(3)$ \\
\hline $\mathrm{C} 2$ & $\mathrm{C} 3$ & $\mathrm{C} 4$ & $126.0(4)$ & $\mathrm{C} 22$ & $\mathrm{C} 21$ & $\mathrm{C} 27$ & $126.1(4)$ \\
\hline
\end{tabular}




$\begin{array}{llllllll}\mathrm{C} 5 & \mathrm{C} 4 & \mathrm{C} 3 & 130.4(4) & \mathrm{C} 21 & \mathrm{C} 22 & \mathrm{C} 23 & 131.2(4) \\ \mathrm{C} 4 & \mathrm{C} 5 & \mathrm{C} 6 & 131.4(4) & \mathrm{C} 24 & \mathrm{C} 23 & \mathrm{C} 22 & 128.9(4) \\ \mathrm{N} 2 & \mathrm{C} 6 & \mathrm{C} 5 & 126.4(3) & \mathrm{C} 23 & \mathrm{C} 24 & \mathrm{C} 25 & 126.7(4) \\ \mathrm{N} 2 & \mathrm{C} 6 & \mathrm{C} 7 & 109.7(3) & \mathrm{C} 26 & \mathrm{C} 25 & \mathrm{C} 24 & 130.2(4) \\ \mathrm{C} 5 & \mathrm{C} 6 & \mathrm{C} 7 & 123.9(3) & \mathrm{C} 25 & \mathrm{C} 26 & \mathrm{C} 27 & 130.1(4) \\ \mathrm{O} 2 & \mathrm{C} 7 & \mathrm{C} 1 & 118.1(3) & \mathrm{N} 3 & \mathrm{C} 27 & \mathrm{C} 21 & 109.3(3) \\ \mathrm{O} 2 & \mathrm{C} 7 & \mathrm{C} 6 & 114.8(3) & \mathrm{N} 3 & \mathrm{C} 27 & \mathrm{C} 26 & 125.8(3) \\ \mathrm{C} 1 & \mathrm{C} 7 & \mathrm{C} 6 & 127.0(4) & \mathrm{C} 26 & \mathrm{C} 27 & \mathrm{C} 21 & 124.6(3) \\ \mathrm{C} 9 & \mathrm{C} 8 & \mathrm{~N} 2 & 128.1(3) & \mathrm{N} 3 & \mathrm{C} 28 & \mathrm{C} 33 & 112.6(3) \\ \mathrm{C} 9 & \mathrm{C} 8 & \mathrm{C} 13 & 119.4(3) & \mathrm{C} 29 & \mathrm{C} 28 & \mathrm{~N} 3 & 127.5(3) \\ \mathrm{C} 13 & \mathrm{C} 8 & \mathrm{~N} 2 & 112.3(3) & \mathrm{C} 29 & \mathrm{C} 28 & \mathrm{C} 33 & 119.6(3) \\ \mathrm{C} 10 & \mathrm{C} 9 & \mathrm{C} 8 & 119.8(4) & \mathrm{C} 28 & \mathrm{C} 29 & \mathrm{C} 30 & 120.1(4) \\ \mathrm{C} 11 & \mathrm{C} 10 & \mathrm{C} 9 & 120.8(4) & \mathrm{C} 31 & \mathrm{C} 30 & \mathrm{C} 29 & 120.2(4) \\ \mathrm{C} 10 & \mathrm{C} 11 & \mathrm{C} 12 & 120.1(3) & \mathrm{C} 30 & \mathrm{C} 31 & \mathrm{C} 32 & 120.5(4) \\ \mathrm{C} 13 & \mathrm{C} 12 & \mathrm{C} 11 & 120.4(4) & \mathrm{C} 33 & \mathrm{C} 32 & \mathrm{C} 31 & 120.3(4) \\ \mathrm{N} 1 & \mathrm{C} 13 & \mathrm{C} 8 & 112.7(3) & \mathrm{N} 4 & \mathrm{C} 33 & \mathrm{C} 28 & 112.2(3) \\ \mathrm{C} 12 & \mathrm{C} 13 & \mathrm{~N} 1 & 127.6(3) & \mathrm{C} 32 & \mathrm{C} 33 & \mathrm{~N} 4 & 128.3(3) \\ \mathrm{C} 12 & \mathrm{C} 13 & \mathrm{C} 8 & 119.6(3) & \mathrm{C} 32 & \mathrm{C} 33 & \mathrm{C} 28 & 119.2(3) \\ \mathrm{N} 1 & \mathrm{C} 14 & \mathrm{C} 15 & 126.2(3) & \mathrm{N} 4 & \mathrm{C} 34 & \mathrm{C} 35 & 125.2(3) \\ \mathrm{N} 1 & \mathrm{C} 14 & \mathrm{C} 20 & 109.5(3) & \mathrm{N} 4 & \mathrm{C} 34 & \mathrm{C} 40 & 110.3(3) \\ \mathrm{C} 15 & \mathrm{C} 14 & \mathrm{C} 20 & 124.0(3) & \mathrm{C} 35 & \mathrm{C} 34 & \mathrm{C} 40 & 124.3(3) \\ \mathrm{C} 16 & \mathrm{C} 15 & \mathrm{C} 14 & 130.0(4) & \mathrm{C} 36 & \mathrm{C} 35 & \mathrm{C} 34 & 130.3(4) \\ \mathrm{C} 15 & \mathrm{C} 16 & \mathrm{C} 17 & 130.7(4) & \mathrm{C} 35 & \mathrm{C} 36 & \mathrm{C} 37 & 130.6(4) \\ \mathrm{C} 18 & \mathrm{C} 17 & \mathrm{C} 16 & 126.2(4) & \mathrm{C} 38 & \mathrm{C} 37 & \mathrm{C} 36 & 126.4(4) \\ \mathrm{C} 17 & \mathrm{C} 18 & \mathrm{C} 19 & 129.4(4) & \mathrm{C} 37 & \mathrm{C} 38 & \mathrm{C} 39 & 129.7(4) \\ \mathrm{C} 20 & \mathrm{C} 19 & \mathrm{C} 18 & 130.5(4) & \mathrm{C} 40 & \mathrm{C} 39 & \mathrm{C} 38 & 130.6(4) \\ \mathrm{O} 1 & \mathrm{C} 20 & \mathrm{C} 14 & 114.8(3) & \mathrm{O} 3 & \mathrm{C} 40 & \mathrm{C} 34 & 114.4(3) \\ \mathrm{O} 1 & \mathrm{C} 20 & \mathrm{C} 19 & 118.3(3) & \mathrm{O} 3 & \mathrm{C} 40 & \mathrm{C} 39 & 117.9(3) \\ \mathrm{C} 19 & \mathrm{C} 20 & \mathrm{C} 14 & 126.9(3) & \mathrm{C} 39 & \mathrm{C} 40 & \mathrm{C} 34 & 127.7(3)\end{array}$

Table S20. Hydrogen Atom Coordinates $\left(\AA \times 10^{4}\right)$ and Isotropic Displacement Parameters $\left(\AA^{2} \times 10^{3}\right)$ for $A l^{\mathrm{IIII}}(\operatorname{tralen}) \mathrm{Cl}$.

\begin{tabular}{lllll}
\multicolumn{1}{c}{ Atom } & \multicolumn{2}{c}{$\boldsymbol{y}$} & & $\boldsymbol{z}$ \\
H1 & 6589.56 & 10327.29 & 2764.07 & 32 \\
H2 & 6219.56 & 11730.96 & 3187.62 & 39 \\
H3 & 5900.75 & 12039.8 & 4252.07 & 40 \\
H4 & 5779.59 & 10932.04 & 5111.51 & 38 \\
H5 & 6036.38 & 9356.1 & 5174.03 & 32 \\
H9 & 6726.43 & 8320.7 & 5929.41 & 30 \\
H10 & 6657.54 & 7052.72 & 6768.98 & 34 \\
H11 & 6516.72 & 5415.52 & 6362.51 & 32
\end{tabular}




$\begin{array}{lllll}\text { H12 } & 6427.29 & 5020.46 & 5098.3 & 29 \\ \text { H15 } & 5464.85 & 4754.49 & 3984.15 & 30 \\ \text { H16 } & 5098.48 & 3411.98 & 3295.82 & 34 \\ \text { H17 } & 5470.56 & 2959.6 & 2269.57 & 34 \\ \text { H18 } & 6033.48 & 5926.53 & 1561.31 & 34 \\ \text { H19 } & 6384.09 & 4838.94 & 1707.19 & 30 \\ \text { H22 } & 8668.06 & 6418.16 & 8330.35 & 29 \\ \text { H23 } & 9060.28 & 7396.88 & 8502.13 & 32 \\ \text { H24 } & 9596.46 & 6965.26 & 7781.18 & 34 \\ \text { H25 } & 9941.35 & 5617.73 & 6738.43 & 34 \\ \text { H26 } & 5370.63 & 6045.92 & 29 \\ \text { H29 } & 9567.7 & 4981.92 & 4932.04 & 29 \\ \text { H30 } & 8605.46 & 3353.91 & 3661.95 & 34 \\ \text { H31 } & 8501.61 & 2086.25 & 3225.91 & 33 \\ \text { H32 } & 8271.22 & 1045.51 & 4058.39 & 31 \\ \text { H35 } & 8180.98 & -545.53 & 4810.43 & 32 \\ \text { H36 } & 8934.38 & -1666.54 & 4847.78 & 36 \\ \text { H37 } & 9152.09 & -1383.93 & 5671.22 & 36 \\ \text { H38 } & 8971.1 & 10.99 & 6733.13 & 33 \\ \text { H39 } & 8644.37 & & 7181.82 & 31\end{array}$

